Article

\title{
Preparation of Half- and Post-Metallocene Hafnium Complexes with Tetrahydroquinoline and Tetrahydrophenanthroline Frameworks for Olefin Polymerization
}

\author{
Jun Won Baek ${ }^{1}$, Su Jin Kwon ${ }^{1}$, Hyun Ju Lee ${ }^{1}$, Tae Jin Kim ${ }^{1}$, Ji Yeon Ryu ${ }^{2}$, Junseong Lee ${ }^{2}$, \\ Eun Ji Shin ${ }^{3}$, Ki Soo Lee ${ }^{3}$ and Bun Yeoul Lee ${ }^{1, *(D)}$ \\ 1 Department of Molecular Science and Technology, Ajou University, Suwon 16499, Korea \\ 2 Department of Chemistry, Chonnam National University, 77 Yongbong-ro, Buk-gu, Gwangju 500-757, Korea \\ 3 LG Chem, Ltd., 188, Munji-ro, Yuseong-gu Daejeon 305-738, Korea \\ * Correspondence: bunyeoul@ajou.ac.kr; Tel.: +82-31-219-1844
}

Received: 25 May 2019; Accepted: 25 June 2019; Published: 27 June 2019

check for updates

\begin{abstract}
Hafnium complexes have drawn attention for their application as post-metallocene catalysts with unique performance in olefin polymerization. In this work, a series of half-metallocene $\mathrm{HfMe}_{2}$ complexes, bearing a tetrahydroquinoline framework, as well as a series of $\left[\mathrm{N}^{\text {amido }}, \mathrm{N}, \mathrm{C}^{\text {aryl }}\right] \mathrm{HfMe}_{2}$-type post-metallocene complexes, bearing a tetrahydrophenanthroline framework, were prepared; the structures of the prepared $\mathrm{Hf}$ complexes were unambiguously confirmed by X-ray crystallography. When the prepared complexes were reacted with anhydrous $\left[\left(\mathrm{C}_{18} \mathrm{H}_{37}\right)_{2} \mathrm{~N}(\mathrm{H}) \mathrm{Me}\right]^{+}\left[\mathrm{B}\left(\mathrm{C}_{6} \mathrm{~F}_{5}\right)_{4}\right]^{-}$, desired ion-pair complexes, in which $\left(\mathrm{C}_{18} \mathrm{H}_{37}\right)_{2} \mathrm{NMe}$ coordinated to the Hf center, were cleanly afforded. The activated complexes generated from the half-metallocene complexes were inactive for the copolymerization of ethylene/propylene, while those generated from post-metallocene complexes were active. Complex bearing bulky isopropyl substituents (12) exhibited the highest activity. However, the activity was approximately half that of the prototype pyridylamido-Hf Dow catalyst. The comonomer incorporation capability was also inferior to that of the pyridylamido-Hf Dow catalyst. However, 12 performed well in the coordinative chain transfer polymerization performed in the presence of (octyl $)_{2} \mathrm{Zn}$, converting all the fed (octyl $)_{2} \mathrm{Zn}$ to (polyolefinyl ${ }_{2} \mathrm{Zn}$ with controlled lengths of the polyolefinyl chain.
\end{abstract}

Keywords: post-metallocene; half-metallocene; hafnium complex; olefin polymerization; coordinative chain transfer polymerization

\section{Introduction}

Polyolefins (POs) are the most abundant polymers, which are mostly produced using the Ziegler-Natta catalyst. The conventional heterogeneous multi-site Ziegler-Natta catalyst has been replaced with homogeneous single-site catalysts, although the former is still a main player in the production of POs. The use of homogeneous single-site catalysts originated with the serendipitous discovery of methylaluminoxane (MAO) by Kaminsky [1]. The initial Zr-based metallocene catalysts, Ti-based half-metallocenes, and post-metallocenes with non-cyclopentadienyl ligands, were developed successively (Scheme 1) [2,3]. A typical example of half-metallocenes is $\left[\mathrm{Me}_{2} \mathrm{Si}\left(\eta^{5}-\mathrm{Me}_{4} \mathrm{C}_{5}\right)\left(\mathrm{N}^{t} \mathrm{Bu}\right)\right] \mathrm{TiCl}{ }_{2}$, which was discovered in the early 1990s at Dow (II Scheme 1) [4]. The Ti-based half-metallocenes characteristically exhibit, similar to the Zr-based metallocene catalysts, higher $\alpha$-olefin incorporation in ethylene/ $\alpha$-olefin copolymerizations, which enables the commercial production of polyolefin elastomers (POE). A typical example of post-metallocenes is the pyridylamido-Hf complex (III in 
Scheme 1), which was discovered in the early 2000s also at Dow $[5,6]$. The pyridylamido-Hf complex exhibits excellent $\alpha$-olefin incorporation capability [7], and is capable of controlling the tacticity in the propylene polymerization to produce isotactic-polypropylene [8-10]. A unique characteristic of III is that the $\beta$-elimination process-an intrinsic chain transfer process that inevitably occurs during the olefin polymerization performed with the conventional Zr-based metallocene and Ti-based half metallocene-can be completely prevented [11]. The DFT (density functional theory) calculation results suggest that the $\beta$-hydrogen transfer reaction is disfavored by the absence of agostic hydrogen interactions, due to the less acidic nature of the hafnium center $[9,10]$. In contrast, the agostic hydrogen interaction plays a significant role in the typical Zr-based metallocene catalysis [12]. Absence of the $\beta$-elimination process enables the construction of high-molecular-weight polyolefin chains with various block compositions [13]. When polymerization is performed in the presence of a dialkylzinc (e.g., $\mathrm{Et}_{2} \mathrm{Zn}$ ), polymer chains are uniformly grown from dialkylzinc due to the rapid alkyl exchange between $\mathrm{Zn}$ and the Hf sites; this is called the coordinative chain transfer polymerization (CCTP) [14-16]. CCTP is judiciously utilized in the commercial production of olefin block copolymer (OBC) at Dow [5,17-19]. It was also demonstrated that the CCTP involving III, could be switched to anionic styrene polymerization to prepare polyolefin-polystyrene block copolymers [11,20-22]. In this context, many thorough studies have been performed to detail III, and to improve the catalytic activity by modifying its ligand skeleton [23-32]. To develop an upgraded catalyst relative to III, we prepared various $\mathrm{Hf}$ complexes. Hafnium catalysts typically exhibit low $\alpha$-olefin incorporation in ethylene/ $\alpha$-olefin copolymerizations and, in this work, ligands were designed to minimize steric hindrance around the reaction site.
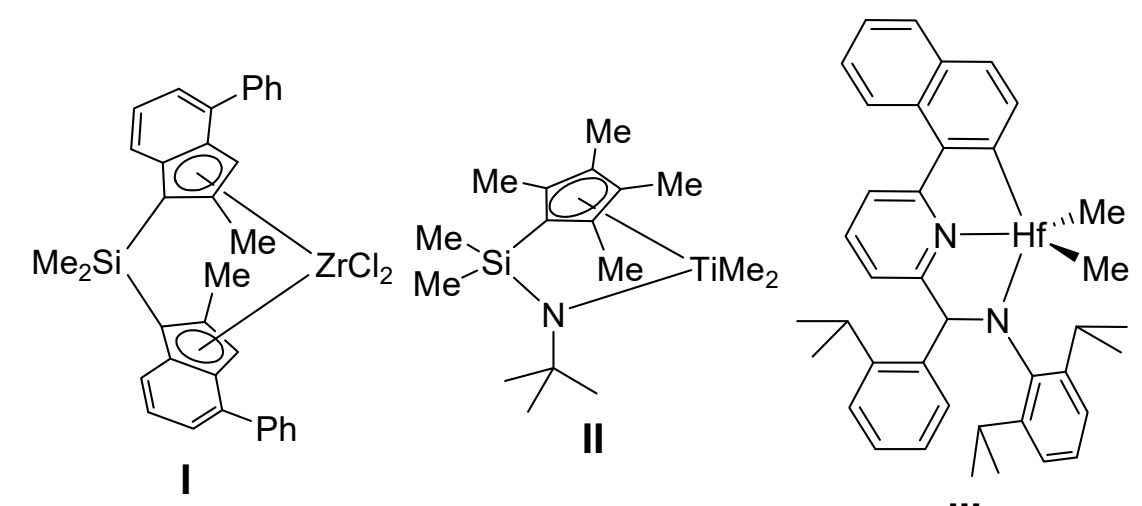

III

Scheme 1. Typical examples of metallocene, half-metallocene, and post-metallocene catalysts.

\section{Materials and Methods}

All the experiments were performed in an inert atmosphere using a standard glove box and Schlenk techniques. Toluene, hexane, and THF were distilled from benzophenone ketyl. Methylcyclohexane (anhydrous grade) used for the polymerization reactions was purchased from Tokyo Chemical Industry (TCI, Tokyo, Japan) and purified over a Na/K alloy. Sublimed-grade $\mathrm{HfCl}_{4}$ was purchased from Strem Chemicals (Newburyport, MA, USA) and used as received. The ethylene/propylene mixed gas was purified over trioctylaluminum $\left(0.6 \mathrm{M}\right.$ in methylcyclohexane), in a bomb reactor $(2.0 \mathrm{~L})$. The ${ }^{1} \mathrm{H} \mathrm{NMR}$ (600 MHz) and ${ }^{13} \mathrm{C}$ NMR (150 MHz) analyses were performed on a JEOL ECZ 600 instrument (Tokyo, Japan). Elemental analyses were performed at the Analytical Center of Ajou University (Suwon, South Korea). The GPC data were obtained in 1,2,4-trichlorobenzene, at $160{ }^{\circ} \mathrm{C}$, using a PL-GPC 220 system equipped with a RI detector and two columns (PLgel mixed-B $7.5 \times 300 \mathrm{~mm}$ from Varian (Polymer Lab, Palo Alto, CA, USA)). The ligand precursors for 1-6 [33,34] and compounds 7-8 [35] were prepared according to the reported method. 


\subsection{Preparation of 1}

$\operatorname{MeMgBr}(2.60 \mathrm{~mL}, 3.11 \mathrm{M}$ in diethyl ether) was added dropwise to a stirred solution of 8-(tetramethylcyclopentadienyl)-1,2,3,4-tetrahydroquinoline $(0.500 \mathrm{~g}, 1.97 \mathrm{mmol})$ and THF $(7 \mathrm{~mL})$ at room temperature. The solution was stirred for $6 \mathrm{~h}$ at $60^{\circ} \mathrm{C}$, and the generated methane gas was vented off, simultaneously. After cooling to room temperature, $\mathrm{HfCl}_{4}(0.632 \mathrm{~g}, 1.97 \mathrm{mmol})$ was added to the resulting solution. After the solution was stirred for $12 \mathrm{~h}$ at room temperature, the solvent was removed using a vacuum line. The residue was extracted with hexane $(4 \mathrm{~mL} \times 6)$. The removal of the solvent produced a yellow solid, which was pure according to the results of the ${ }^{1} \mathrm{H}$ and ${ }^{13} \mathrm{C} N \mathrm{NR}$ spectra analyses (Figure S1 in Supporting Information; 0.794 g, 87\%). An analytical pure compound, containing single crystals that are suitable for $\mathrm{X}$-ray crystallography, was obtained by recrystallization in hexane at $-30{ }^{\circ} \mathrm{C} .{ }^{1} \mathrm{H}$ NMR $\left(\mathrm{C}_{6} \mathrm{D}_{6}\right): 87.05(\mathrm{~d}, J=7.8 \mathrm{~Hz}, 1 \mathrm{H}), 6.96(\mathrm{~d}, J=6.6 \mathrm{~Hz}, 1 \mathrm{H}), 6.82(\mathrm{t}, J=7.2 \mathrm{~Hz}$, $1 \mathrm{H}), 3.80(\mathrm{t}, J=4.8 \mathrm{~Hz}, 2 \mathrm{H}), 2.50(\mathrm{t}, J=6.6 \mathrm{~Hz}, 2 \mathrm{H}), 2.00(\mathrm{~s}, 6 \mathrm{H}), 1.78(\mathrm{~s}, 6 \mathrm{H}), 1.62(\mathrm{q}, J=6 \mathrm{~Hz}, 2 \mathrm{H}),-0.02$ (s, $\left.\mathrm{Hf}\left(\mathrm{CH}_{3}\right)_{2} 6 \mathrm{H}\right) \mathrm{ppm} .{ }^{13} \mathrm{C}\left\{{ }^{1} \mathrm{H}\right\} \mathrm{NMR}\left(\mathrm{C}_{6} \mathrm{D}_{6}\right): \delta 10.80,22.94,27.19,43.31,48.35,116.67,118.73,122.10$, 125.57, 129.17, 130.78, 160.69 ppm. Anal. Calcd. $\left(\mathrm{C}_{20} \mathrm{H}_{27} \mathrm{HfN}\right): \mathrm{C}, 52.23 ; \mathrm{H}, 5.92 ; \mathrm{N}, 3.05 \%$. Found: $\mathrm{C}$, $52.21 ; \mathrm{H}, 5.91 ; \mathrm{N}, 3.08 \%$.

\subsection{Preparation of 2}

Complex 2 was prepared by the same procedure and experimental conditions as those employed for 1, using 2-methyl-8-(tetramethylcyclopentadienyl)-1,2,3,4-tetrahydroquinoline (0.420 g, $1.57 \mathrm{mmol})$. The reaction between the ligand precursor and $\mathrm{MeMgBr}$ was so slow that $30 \mathrm{~h}$ was required for the reaction to reach completion. A yellow solid compound was obtained $(0.594 \mathrm{~g}, 80 \%) .{ }^{1} \mathrm{H} \mathrm{NMR}\left(\mathrm{C}_{6} \mathrm{D}_{6}\right)$ : $\delta 7.06(\mathrm{~d}, J=7.2 \mathrm{~Hz}, 1 \mathrm{H}), 7.00(\mathrm{~d}, J=7.0 \mathrm{~Hz}, 1 \mathrm{H}), 6.82(\mathrm{t}, J=6.6 \mathrm{~Hz} 1 \mathrm{H}), 4.15(\mathrm{~m}, 1 \mathrm{H}, \mathrm{NCH}), 2.64(\mathrm{~m}, 1 \mathrm{H})$, $2.47(\mathrm{t}, J=5.4 \mathrm{~Hz}, 1 \mathrm{H}), 2.00\left(\mathrm{~s}, 6 \mathrm{H}, \mathrm{CH}_{3}\right), 1.80\left(\mathrm{~s}, 3 \mathrm{H}, \mathrm{CH}_{3}\right), 1.75\left(\mathrm{~s}, 3 \mathrm{H}, \mathrm{CH}_{3}\right), 1.48-1.56(\mathrm{~m}, 2 \mathrm{H}), 1.32$ $\left(\mathrm{d}, J=6.0 \mathrm{~Hz}, 3 \mathrm{H}, \mathrm{NCCH}_{3}\right),-15.2\left(\mathrm{~s}, 3 \mathrm{H}, \mathrm{HfCH}_{3}\right),-27.8\left(\mathrm{~s}, 3 \mathrm{H}, \mathrm{HfCH}_{3}\right)$ ppm. $13 \mathrm{C}\{1 \mathrm{H}\} \mathrm{NMR}\left(\mathrm{C}_{6} \mathrm{D}_{6}\right)$ : $\delta 10.66,10.87,10.88,11.01,21.50,23.68,29.08,47.40,47.52,49.83,116.31,117.70,118.55,121.32,125.51$, 129.28, 130.15, 130.32, 159.54 ppm. Anal. Calcd. $\left(\mathrm{C}_{21} \mathrm{H}_{29} \mathrm{HfN}\right)$ : $\mathrm{C}, 53.22 ; \mathrm{H}, 6.17 ; \mathrm{N}, 2.96 \%$. Found: $\mathrm{C}$, $53.22 ; \mathrm{H}, 6.14 ; \mathrm{N}, 2.98 \%$.

\subsection{Preparation of 3}

Complex 3 was prepared by the same procedure and experimental conditions as those employed for 1, using 8-(2,4,5-trimethyl-6H-cyclopenta[b]thiophen-6-yl)-1,2,3,4-tetrahydroquinoline (0.420 g, $1.36 \mathrm{mmol}$ ). A light brown solid compound was obtained. An analytical pure compound, containing single crystals that are suitable for $\mathrm{X}$-ray crystallography, was obtained by recrystallization in hexane at $-30{ }^{\circ} \mathrm{C}(0.530 \mathrm{~g}, 62 \%) .{ }^{1} \mathrm{H}$ NMR $\left(\mathrm{C}_{6} \mathrm{D}_{6}\right): \delta 7.15(\mathrm{dd}, J=6.6 \mathrm{~Hz}, 1 \mathrm{H}), 6.95(\mathrm{dd}, J=7.8 \mathrm{~Hz}, 1 \mathrm{H}), 6.78$ $(\mathrm{t}, J=6.6 \mathrm{~Hz}, 1 \mathrm{H}), 6.41$ (quartet, $J=1.2 \mathrm{~Hz}, 1 \mathrm{H}, \mathrm{SCCH}), 3.80(\mathrm{~m}, 1 \mathrm{H}), 3.75(\mathrm{~m}, 1 \mathrm{H}), 2.46(\mathrm{t}, J=6.0 \mathrm{~Hz}$, $2 \mathrm{H}), 2.20\left(\mathrm{~s}, 3 \mathrm{H}, \mathrm{CH}_{3}\right), 2.10\left(\mathrm{~d}, J=1.8 \mathrm{~Hz}, 3 \mathrm{H}, \mathrm{SCCH}_{3}\right), 1.60(\mathrm{~m}, 2 \mathrm{H}), 0.07\left(\mathrm{~s}, 3 \mathrm{H}, \mathrm{HfCH}_{3}\right),-0.29$ (s, $\left.3 \mathrm{H}, \mathrm{HfCH}_{3}\right)$ ppm. ${ }^{13} \mathrm{C}\left\{{ }^{1} \mathrm{H}\right\} \mathrm{NMR}\left(\mathrm{C}_{6} \mathrm{D}_{6}\right): \delta 11.30,11.58,16.21,22.73,27.12,43.13,49.72,50.53,106.21$, 116.51, 117.20, 118.80, 122.42, 124.57, 129.77, 129.94, 134.89, 136.11, 143.96, 160.81 ppm. Anal. Calcd. $\left(\mathrm{C}_{21} \mathrm{H}_{25} \mathrm{HfNS}\right): \mathrm{C}, 50.25 ; \mathrm{H}, 5.02 ; \mathrm{N}, 2.79 ; \mathrm{S}, 6.39 \%$. Found: $\mathrm{C}, 50.21 ; \mathrm{H}, 5.01 ; \mathrm{N}, 2.78 ; \mathrm{S}, 6.39 \%$.

\subsection{Preparation of 4}

Complex 4 was prepared by the same procedure and experimental conditions as those employed for 1, using 2-methyl-8-(2,4,5-trimethyl-6H-cyclopenta[b]thiophen-6-yl)-1,2,3,4-tetrahydroquinoline $(0.420 \mathrm{~g}, 1.36 \mathrm{mmol})$. The reaction between the ligand precursor and $\mathrm{MeMgBr}$ was so slow that $30 \mathrm{~h}$ was required for the reaction to reach completion. A light brown solid compound was obtained. An analytically pure compound was obtained through recrystallization in hexane at $-30{ }^{\circ} \mathrm{C}(0.467 \mathrm{~g}$, 67\%). ${ }^{1} \mathrm{H}$ NMR $\left(\mathrm{C}_{6} \mathrm{D}_{6}\right): \delta 7.17$ and $7.15(\mathrm{~d}, J=6.3 \mathrm{~Hz}, 1 \mathrm{H}), 6.99$ and $6.98(\mathrm{~d}, J=8.4 \mathrm{~Hz}, 1 \mathrm{H}), 6.79(\mathrm{t}$, $J=7.8 \mathrm{~Hz}, 1 \mathrm{H}), 6.42$ and $6.38(\mathrm{~d}, J=1.2 \mathrm{~Hz}, 1 \mathrm{H}, \mathrm{SCCH}), 4.21$ and $4.14(\mathrm{~m}, 1 \mathrm{H}, \mathrm{NCH}), 2.66$ and 2.63 $(\mathrm{m}, 1 \mathrm{H}), 2.44$ and $2.41(\mathrm{dt}, J=4.8 \mathrm{~Hz}, \mathrm{H}), 2.21$ and $2.20\left(\mathrm{~s}, 3 \mathrm{H}, \mathrm{CH}_{3}\right), 2.12$ and $2.11\left(\mathrm{~s}, 3 \mathrm{H}, \mathrm{SCCH}_{3}\right)$, 
1.85 and $1.79\left(\mathrm{~s}, 3 \mathrm{H}, \mathrm{CH}_{3}\right), 1.55(\mathrm{~m}, 2 \mathrm{H}), 1.3$ and $1.27\left(\mathrm{~d}, J=7.2 \mathrm{~Hz}, 3 \mathrm{H}, \mathrm{SCCH}_{3}\right), 0.09$ and $0.08(\mathrm{~s}, 3 \mathrm{H}$, $\left.\mathrm{HfCH}_{3}\right),-0.28$ and $\left.-0.30\left(\mathrm{~s}, 3 \mathrm{H}, \mathrm{HfCH}_{3}\right) \mathrm{ppm} .{ }^{13} \mathrm{C}_{\{}{ }^{1} \mathrm{H}\right\}$ NMR $\left(\mathrm{C}_{6} \mathrm{D}_{6}\right): \delta 11.12,11.41,11.58,11.76,16.12$, 16.23, 21.20, 21.35, 23.07, 23.54, 28.50, 28.73, 46.63, 47.07, 48.71, 49.69, 51.34, 51.71, 105.88, 107.45, 116.47, $116.59,116.85,118.62,121.41,121.65,124.63,129.12,129.83,129.92,131.20,134.42,134.67,135.29,135.68$, 143.86, 144.41 159.47, 159.61 ppm. Anal. Calcd. ( $\left.\mathrm{C}_{22} \mathrm{H}_{27} \mathrm{HfNS}\right)$ : $\mathrm{C}, 51.21 ; \mathrm{H}, 5.27 ; \mathrm{N}, 2.71 ; \mathrm{S}, 6.21 \%$. Found: C, 51.20; H, 5.24; N, 2.73; S, 6.22\%.

\subsection{Preparation of 5}

Complex $\mathbf{5}$ was prepared by the same procedure and experimental conditions as those employed for 1 , using fluorenyltetrahydroquinoline $(0.194 \mathrm{~g}, 0.651 \mathrm{mmol})$. The product was marginally soluble in hexane and it was extracted with toluene $(2 \mathrm{~mL} \times 3)$. An analytically pure compound was obtained through recrystallization in hexane, at $-30{ }^{\circ} \mathrm{C}(0.161 \mathrm{~g}, 49 \%) .{ }^{1} \mathrm{H} N M R\left(\mathrm{C}_{6} \mathrm{D}_{6}\right): \delta 7.88(\mathrm{~d}, J=8.4 \mathrm{~Hz}$, $2 \mathrm{H}), 7.34(\mathrm{~d}, J=6.6 \mathrm{~Hz}, 1 \mathrm{H}), 7.22(\mathrm{~d}, J=8.4 \mathrm{~Hz}, 2 \mathrm{H}), 7.01(\mathrm{t}, J=7.2 \mathrm{~Hz}, 2 \mathrm{H}), 6.94(\mathrm{t}, J=8.4 \mathrm{~Hz}, 2 \mathrm{H}), 6.91$ $(\mathrm{t}, J=7.2 \mathrm{~Hz}, 1 \mathrm{H}), 3.60\left(\mathrm{~m}, 2 \mathrm{H}, \mathrm{NCH}_{2}\right), 2.53(\mathrm{t}, J=6.0 \mathrm{~Hz}, 2 \mathrm{H}) 1.56(\mathrm{~m}, 2 \mathrm{H}),-0.69\left(\mathrm{~s}, 6 \mathrm{H}, \mathrm{Hf}\left(\mathrm{CH}_{3}\right)_{2}\right)$ ppm. ${ }^{13} \mathrm{C}\left\{{ }^{1} \mathrm{H}\right\} \operatorname{NMR}\left(\mathrm{C}_{6} \mathrm{D}_{6}\right): \delta 22.51,27.22,42.89,52.53,103.54,114.32,118.91,121.16,121.84,122.58$, $123.58,125.13,128.60,129.88,130.24,136.63,159.98$ ppm. Anal. Calcd. $\left(\mathrm{C}_{24} \mathrm{H}_{23} \mathrm{HfN}\right): \mathrm{C}, 57.20 ; \mathrm{H}, 4.60$; N, 2.78\%. Found: C, 57.16; H, 4.59; N, 2.79\%.

\subsection{Preparation of 6}

Complex 6 was prepared by the same procedure and experimental conditions as those employed for 1, using 2-methyl-8-(fluorenyl)tetrahydroquinoline $(0.154 \mathrm{~g}, 0.494 \mathrm{mmol})$. The product was marginally soluble in hexane and it was extracted with toluene $(2 \mathrm{~mL} \times 3)$. An analytical pure compound, containing single crystals that are suitable for X-ray crystallography was obtained by recrystallization in hexane at $-30{ }^{\circ} \mathrm{C}(0.158 \mathrm{~g}, 62 \%) .{ }^{1} \mathrm{H}$ NMR $\left(\mathrm{C}_{6} \mathrm{D}_{6}\right): \delta 7.88(\mathrm{~d}, J=8.4 \mathrm{~Hz}, \mathrm{H}), 7.86(\mathrm{~d}$, $J=8.4 \mathrm{~Hz}, \mathrm{H}), 7.34(\mathrm{~d}, J=7.2 \mathrm{~Hz}, \mathrm{H}), 7.24(\mathrm{~d}, J=9.0 \mathrm{~Hz}, \mathrm{H}), 7.18(\mathrm{~d}, J=8.4 \mathrm{~Hz}, \mathrm{H}), 7.12(\mathrm{~d}, J=7.8 \mathrm{~Hz}, \mathrm{H})$, $7.05(\mathrm{t}, J=7.8 \mathrm{~Hz}, \mathrm{H}), 7.01(\mathrm{t}, J=7.2 \mathrm{~Hz}, \mathrm{H}), 6.93(\mathrm{~m}, 3 \mathrm{H}), 4.01(\mathrm{~m}, \mathrm{H}), 2.73(\mathrm{~m}, \mathrm{H}), 2.48(\mathrm{dt}, J=4.8 \mathrm{~Hz}$, $\mathrm{H}), 1.52(\mathrm{~m}, 2 \mathrm{H}), 1.09\left(\mathrm{~d}, J=6.6 \mathrm{~Hz}, 3 \mathrm{H}, \mathrm{NCCH}_{3}\right),-0.68\left(\mathrm{~s}, 3 \mathrm{H}, \mathrm{HfCH}_{3}\right),-0.71\left(\mathrm{~s}, 3 \mathrm{H}, \mathrm{HfCH}_{3}\right) \mathrm{ppm}$. ${ }^{13} \mathrm{C}\left\{{ }^{1} \mathrm{H}\right\}$ NMR $\left(\mathrm{C}_{6} \mathrm{D}_{6}\right): \delta 20.78,22.89,28.05,45.96,51.32,54.09,103.13,113.66,115.63,118.80,120.85$, 120.90, 121.48, 122.42, 122.94, 123.68, 124.86, 125.54, 128.70, 129.99, 130.30, 135.85, 136.20, 158.46 ppm. Anal. Calcd. $\left(\mathrm{C}_{25} \mathrm{H}_{25} \mathrm{HfN}\right)$ : C, 57.97; H, 4.87; N, 2.70\%. Found: C, 57.89; H, 4.79; N, 2.73\%.

\subsection{Preparation of 9}

Isopropyllithium (6.13 $\mathrm{mL}, 0.70 \mathrm{M}$ in pentane) was slowly added to a stirred suspension of 2-phenyl-1,10-phenanthroline $(1.00 \mathrm{~g}, 3.90 \mathrm{mmol})$ in toluene $(10 \mathrm{~mL})$, at $-30{ }^{\circ} \mathrm{C}$. The solution was heated to $0^{\circ} \mathrm{C}$ and stirred for $35 \mathrm{~min} . \mathrm{H}_{2} \mathrm{O}(16 \mathrm{~mL})$ was added and the organic compounds were extracted with $\mathrm{CH}_{2} \mathrm{Cl}_{2}(3 \times 10 \mathrm{~mL})$. The solvent was removed using a vacuum line and the residue redissolved in $\mathrm{CH}_{2} \mathrm{Cl}_{2}(10 \mathrm{~mL})$. Activated $\mathrm{MnO}_{2}(3.68 \mathrm{~g}, 42.3 \mathrm{mmol})$ was added, and the solution was stirred for $12 \mathrm{~h}$ under atmospheric exposure. After filtration over anhydrous $\mathrm{MgSO}_{4}$, the solvent was removed with a rotary evaporator. Pure 2-isopropyl-9-phenyl-1,10-phenanthroline was obtained by column chromatography on silica gel, using ethyl acetate/hexane $(1 / 10, v / v)(1.01 \mathrm{~g}, 87 \%)$. The prepared 2-isopropyl-9-phenyl-1,10-phenanthroline (1.01 g, $3.39 \mathrm{mmol}), \mathrm{Ru}(\mathrm{OTf})(\mathrm{TsDPEN})\left(\eta^{6}\right.$-p-cymene) (TfO = trifluoromethanesulfonate, $\mathrm{TsDPEN}=N$-toluenesulfonyl-1,2-diphenylethylenediamine $)$ ( $0.17 \mathrm{mmol})$, and $\mathrm{MeOH}(17 \mathrm{~mL})$ were added to a bomb reactor. After charging $\mathrm{H}_{2}$ to 50 bar, the reaction mixture was stirred for $12 \mathrm{~h}$ at room temperature. After releasing $\mathrm{H}_{2}$, it was further stirred under atmospheric exposure for $12 \mathrm{~h}$. The solvent was removed with a rotary evaporator and the residue was purified by column chromatography on silica gel, using ethyl acetate/hexane $(1 / 50, v / v)$. A light yellow solid compound was obtained $(0.615 \mathrm{~g}, 60 \%) .{ }^{1} \mathrm{H} \mathrm{NMR}\left(\mathrm{CDCl}_{3}\right): \delta 8.17(\mathrm{~d}, J=6.6 \mathrm{~Hz}$, $2 \mathrm{H}), 8.07(\mathrm{~d}, J=9.0 \mathrm{~Hz}, \mathrm{H}), 7.80(\mathrm{~d}, J=8.4 \mathrm{~Hz}, \mathrm{H}), 7.52(\mathrm{t}, J=7.2 \mathrm{~Hz}, 2 \mathrm{H}), 7.45(\mathrm{t}, J=7.8 \mathrm{~Hz}, \mathrm{H}), 7.15$ $(\mathrm{d}, J=7.2 \mathrm{~Hz}, \mathrm{H}), 6.98(\mathrm{~d}, J=8.4 \mathrm{~Hz}, \mathrm{H}), 6.26(\mathrm{~s}, \mathrm{H}, \mathrm{NH}), 3.25(\mathrm{~m}, \mathrm{H}, \mathrm{NCH}), 2.99(\mathrm{~m}, \mathrm{H}), 2.90(\mathrm{~m}$, $\mathrm{H}), 2.09(\mathrm{~m}, \mathrm{H}), 1.91(\mathrm{~m}, \mathrm{H}), 1.84(\mathrm{~m}, 2 \mathrm{H}, \mathrm{NCCH}), 1.16$ and $1.09\left(\mathrm{~d}, \mathrm{~J}=6.0 \mathrm{~Hz}, 6 \mathrm{H}, \mathrm{CH}\left(\mathrm{CH}_{3}\right)_{2}\right) \mathrm{ppm}$. 
${ }^{13} \mathrm{C}\left\{{ }^{1} \mathrm{H}\right\}$ NMR $\left(\mathrm{C}_{6} \mathrm{D}_{6}\right): \delta 18.54,18.92,24.70,26.86,32.77,56.80,112.96,116.71,118.23,126.85,127.60$, $128.95,128.99,129.07,136.80,137.74,140.57,141.68,153.70$ ppm. $m / z$ calcd. $\left(\left[\mathrm{M}^{+}\right] \mathrm{C}_{21} \mathrm{H}_{22} \mathrm{~N}_{2}\right) 302.4210$. Found: 302.1785 .

\subsection{Preparation of $\mathbf{1 0}$}

$\mathrm{MeMgBr}(1.24 \mathrm{~mL}, 3.11 \mathrm{M}$ in diethyl ether) was added dropwise to a stirred suspension of $\mathrm{HfCl}_{4}(0.300 \mathrm{~g}, 0.938 \mathrm{mmol})$ in toluene $(8 \mathrm{~mL})$ at $-78{ }^{\circ} \mathrm{C}$. After stirring for $1 \mathrm{~h}$ at a controlled temperature within the range of -40 and $-35{ }^{\circ} \mathrm{C}$, the solution was cooled again to $-78{ }^{\circ} \mathrm{C}$. 2-Phenyl-1,2,3,4-tetrahydro-1,10-phenanthroline $(0.24 \mathrm{~g}, 0.94 \mathrm{mmol})$ in toluene $(4 \mathrm{~mL})$ was added dropwise. The resulting solution was stirred at a controlled temperature within the range of -40 and $-35{ }^{\circ} \mathrm{C}$, for $2 \mathrm{~h}$. Subsequently, it was stirred at room temperature overnight. The solvent was removed using a vacuum line and the residue was extracted with hexane $(60 \mathrm{~mL})$. The removal of the solvent produced a dark red solid compound, which was pure according to the results of the ${ }^{1} \mathrm{H}$ and ${ }^{13} \mathrm{C}$ NMR spectra analyses (Figure S10) $(0.79 \mathrm{~g}, 53 \%)$. An analytical pure compound, containing single crystals that are suitable for X-ray crystallography, was obtained by recrystallization in hexane at $-30{ }^{\circ} \mathrm{C} .{ }^{1} \mathrm{H}$ $\operatorname{NMR}\left(\mathrm{C}_{6} \mathrm{D}_{6}\right): \delta 8.47(\mathrm{~d}, J=6.6 \mathrm{~Hz}, \mathrm{H}), 7.63(\mathrm{~d}, J=7.8 \mathrm{~Hz}, \mathrm{H}), 7.57(\mathrm{~d}, J=9.0 \mathrm{~Hz}, \mathrm{H}), 7.47(\mathrm{t}, J=6.0 \mathrm{~Hz}$, $\mathrm{H}), 7.27(\mathrm{dt}, J=7.8 \mathrm{~Hz}, \mathrm{H}), 7.24(\mathrm{~d}, J=8.4 \mathrm{~Hz}, \mathrm{H}), 7.07(\mathrm{~d}, J=7.2 \mathrm{~Hz}, \mathrm{H}), 6.74(\mathrm{~d}, J=8.4 \mathrm{~Hz}, \mathrm{H}), 3.95(\mathrm{~m}$, $2 \mathrm{H}), 2.62(\mathrm{t}, J=6.6 \mathrm{~Hz}, 2 \mathrm{H}), 1.76(\mathrm{~m}, 2 \mathrm{H}), 0.76\left(\mathrm{~s}, 6 \mathrm{H}, \mathrm{Hf}\left(\mathrm{CH}_{3}\right)_{2}\right) \mathrm{ppm} .{ }^{13} \mathrm{C}\left\{{ }^{1} \mathrm{H}\right\} \operatorname{NMR}\left(\mathrm{C}_{6} \mathrm{D}_{6}\right): \delta 22.82$, 26.84, 45.04, 63.72, 112.33, 115.03, 120.11, 124.29, 126.68, 128.62, 130.11, 130.33, 137.32, 141.01, 141.19, 147.77, 151.00, 163.28, 204.57 ppm. Anal. Calcd. $\left(\mathrm{C}_{20} \mathrm{H}_{20} \mathrm{HfN}_{2}\right): \mathrm{C}, 51.45 ; \mathrm{H}, 4.32 ; \mathrm{N}, 6.00 \%$. Found: $\mathrm{C}$, $51.35 ; \mathrm{H}, 4.31 ; \mathrm{N}, 6.10 \%$.

\subsection{Preparation of $\mathbf{1 1}$}

Complex 11 was prepared by the same procedure and experimental conditions as those employed for 10, using 2-butyl-9-phenyl-1,2,3,4-tetrahydro-1,10-phenanthroline (0.390 g, $1.23 \mathrm{mmol})$. A dark red solid compound was obtained $(0.148 \mathrm{~g}, 86 \%)$. An analytical pure compound, containing single crystals that are suitable for X-ray crystallography, was obtained by recrystallization in hexane at $-30{ }^{\circ} \mathrm{C} .{ }^{1} \mathrm{H}$ $\operatorname{NMR}\left(\mathrm{C}_{6} \mathrm{D}_{6}\right): \delta 8.49(\mathrm{~d}, J=6.6 \mathrm{~Hz}, \mathrm{H}), 7.64(\mathrm{~d}, J=7.2 \mathrm{~Hz}, \mathrm{H}), 7.60(\mathrm{~d}, J=9.0 \mathrm{~Hz}, \mathrm{H}), 7.47(\mathrm{t}, J=7.2 \mathrm{~Hz}$, H), $7.27(\mathrm{~m}, 2 \mathrm{H}), 7.14(\mathrm{~d}, J=8.4 \mathrm{~Hz}, \mathrm{H}), 6.77(\mathrm{~d}, J=7.2 \mathrm{~Hz}, \mathrm{H}), 4.29(\mathrm{~m}, \mathrm{H}, \mathrm{NCH}), 2.83(\mathrm{~m}, \mathrm{H}), 2.52(\mathrm{td}$, $J=4.2 \mathrm{~Hz}, \mathrm{H}), 2.03(\mathrm{~m}, \mathrm{H}), 1.90(\mathrm{~m}, \mathrm{H}), 1.74(\mathrm{~m}, 2 \mathrm{H}), 1.35(\mathrm{~m}, 2 \mathrm{H}), 0.95(\mathrm{t}, J=7.2 \mathrm{~Hz}, 3 \mathrm{H}), 0.85(\mathrm{~s}, 3 \mathrm{H}$, $\left.\mathrm{HfCH}_{3}\right), 0.79$ (s, 3H, $\left.\mathrm{HfCH}_{3}\right)$ ppm. $\left.{ }^{13} \mathrm{C}^{1}{ }^{1} \mathrm{H}\right\}$ NMR $\left(\mathrm{C}_{6} \mathrm{D}_{6}\right): \delta 14.48,22.96,23.20,24.57,28.82,35.37,53.14$, 63.37, 65.49, 112.21, 115.01, 119.45, 124.21, 126.83, 128.49, 130.21, 130.30, 137.63, 141.24, 147.60, 150.43, 163.25, 204.42 ppm. Anal. Calcd. $\left(\mathrm{C}_{24} \mathrm{H}_{28} \mathrm{HfN}_{2}\right)$ : C, 55.12; H, 5.40; N, 5.36\%. Found: C, 54.99; H, 5.41; $\mathrm{N}, 5.27 \%$.

\subsection{Preparation of $\mathbf{1 2}$}

Complex 12 was prepared by the same procedure and conditions as those employed for $\mathbf{1 0}$, using 2-isopropyl-9-phenyl-1,2,3,4-tetrahydro-1,10-phenanthroline $(0.20 \mathrm{~g}, 0.66 \mathrm{mmol})$. A dark red solid compound was obtained $(0.330 \mathrm{~g}, 98 \%)$. An analytical pure compound, containing single crystals that are suitable for $\mathrm{X}$-ray crystallography, was obtained by recrystallization in hexane at $-30{ }^{\circ} \mathrm{C} .{ }^{1} \mathrm{H}$ NMR $\left(\mathrm{C}_{6} \mathrm{D}_{6}\right): \delta 8.50(\mathrm{~d}, J=7.2 \mathrm{~Hz}, \mathrm{H}), 7.64(\mathrm{~d}, J=7.2 \mathrm{~Hz}, \mathrm{H}), 7.60(\mathrm{~d}, J=9.0 \mathrm{~Hz}, \mathrm{H}), 7.46(\mathrm{t}, J=6.6 \mathrm{~Hz}, \mathrm{H})$, $7.26(\mathrm{~m}, 2 \mathrm{H}), 7.10(\mathrm{~d}, J=7.8 \mathrm{~Hz}, \mathrm{H}), 6.75(\mathrm{~d}, J=8.4 \mathrm{~Hz}, \mathrm{H}), 4.00(\mathrm{~m}, \mathrm{H}, \mathrm{NCH}), 2.76(\mathrm{~m}, \mathrm{H}), 2.58(\mathrm{~m}, 2 \mathrm{H})$, $1.84(\mathrm{~m}, \mathrm{H}), 1.68(\mathrm{~m}, \mathrm{H}), 1.74(\mathrm{~m}, 2 \mathrm{H}), 1.05$ and $1.01\left(\mathrm{~d}, J=7.2 \mathrm{~Hz}, 6 \mathrm{H}, \mathrm{CH}\left(\mathrm{CH}_{3}\right)_{2}\right), 0.88\left(\mathrm{~s}, 3 \mathrm{H}, \mathrm{HfCH}_{3}\right)$, $0.78\left(\mathrm{~s}, 3 \mathrm{H}, \mathrm{HfCH}_{3}\right)$ ppm. ${ }^{13} \mathrm{C}\left\{{ }^{1} \mathrm{H}\right\}$ NMR $\left(\mathrm{C}_{6} \mathrm{D}_{6}\right): \delta 18.61,21.19,23.12,25.51,32.68,60.75,63.82,66.81$, 112.40, 114.96, 120.50, 124.15, 126.91, 130.19, 130.31, 140.96, 141.37, 147.36, 150.96, 163.49, 204.30 ppm. Anal. Calcd. $\left(\mathrm{C}_{23} \mathrm{H}_{26} \mathrm{HfN}_{2}\right)$ : C, 54.28; H, 5.15; N, 5.50\%. Found: C, 54.37; H, 5.01; N, 5.47\%.

\subsection{Preparation of $\mathbf{1 3}$}

n-BuLi (1.65 ml, 1.61 M in hexane) was slowly added to a stirred suspension of 2-naphthyl-1,10phenanthroline $(0.741 \mathrm{~g}, 2.42 \mathrm{mmol})$ in toluene $(8 \mathrm{~mL})$ at $-10{ }^{\circ} \mathrm{C}$. After stirring for $3 \mathrm{~h}$ at room 
temperature, degassed $\mathrm{H}_{2} \mathrm{O}(3 \mathrm{~mL})$ was added. An aqueous layer was removed with a syringe under atmospheric $\mathrm{N}_{2}$. The solvent was removed using a vacuum line and the residue was dissolved in degassed ethanol $(15 \mathrm{~mL})$ and THF $(5 \mathrm{~mL})$. The solution was transferred to a bomb reactor, containing $\mathrm{Pd} / \mathrm{C}(0.242 \mathrm{mmol}, 10 \mathrm{~mol} \%)$, under atmospheric $\mathrm{N}_{2}$. After the $\mathrm{H}_{2}$ gas was charged to $5 \mathrm{bar}$, it was stirred for $12 \mathrm{~h}$ at room temperature. The $\mathrm{H}_{2}$ gas was released and the catalyst residue was removed by filtration over Celite. The solvent was removed and the residue was purified by column chromatography on silica gel, using ethyl acetate/hexane $(1 / 3, v / v)$. A light yellow solid compound was obtained $(0.420 \mathrm{~g}, 47 \%) .{ }^{1} \mathrm{H}$ NMR $\left(\mathrm{C}_{6} \mathrm{D}_{6}\right): \delta 8.49(\mathrm{~m}, \mathrm{H}), 7.75(\mathrm{~d}, J=8.4 \mathrm{~Hz}, \mathrm{H}), 7.70(\mathrm{~m}, \mathrm{H}), 7.67$ $(\mathrm{d}, J=7.8 \mathrm{~Hz}, \mathrm{H}), 7.64(\mathrm{~d}, J=7.2 \mathrm{~Hz}, \mathrm{H}), 7.33(\mathrm{~m}, 2 \mathrm{H}), 7.30(\mathrm{~m}, 2 \mathrm{H}), 7.20(\mathrm{~d}, J=7.8 \mathrm{~Hz}, \mathrm{H}), 7.02(\mathrm{~d}$, $J=8.4 \mathrm{~Hz}, \mathrm{H}), 6.37(\mathrm{~s}, \mathrm{H}, \mathrm{NH}), 3.16(\mathrm{~m}, \mathrm{H}, \mathrm{NCH}), 2.82(\mathrm{~m}, \mathrm{H}), 2.73(\mathrm{dt}, J=6.0 \mathrm{~Hz}, \mathrm{H}), 1.79(\mathrm{~m}, \mathrm{H}), 1.57$ $(\mathrm{m}, \mathrm{H}), 1.27(\mathrm{~m}, 2 \mathrm{H}), 1.12(\mathrm{~m}, 4 \mathrm{H}), 0.77\left(\mathrm{~d}, J=7.2 \mathrm{~Hz}, 3 \mathrm{H}, \mathrm{CH}_{3}\right) \mathrm{ppm} .{ }^{13} \mathrm{C}\left\{{ }^{1} \mathrm{H}\right\} \mathrm{NMR}\left(\mathrm{C}_{6} \mathrm{D}_{6}\right): \delta 14.28$, 23.11, 26.64, 27.95, 28.25, 36.44, 51.09, 112.87, 116.54, 122.68, 125.59, 126.08, 126.59, 126.65, 126.78, 128.69, $128.99,129.39,132.24,134.64,136.36,137.68,139.93,141.57,156.03 \mathrm{ppm} . \mathrm{m} / z$ calcd $\left(\left[\mathrm{M}^{+}\right] \mathrm{C}_{26} \mathrm{H}_{26} \mathrm{~N}_{2}\right)$ 366.5100. Found: 366.2094 .

\subsection{Preparation of $\mathbf{1 4}$}

Complex 14 was prepared by the same procedure and experimental conditions as those employed for 13, using isopropyllithium $(0.45 \mathrm{~mL}, 0.36 \mathrm{mmol}, 0.79 \mathrm{M}$ in pentane) and 2-naphthyl1,10-phenanthroline $(0.789 \mathrm{~g}, 2.58 \mathrm{mmol})$. A light yellow, sticky solid was obtained $(0.388 \mathrm{~g}, 43 \%) .{ }^{1} \mathrm{H}$ NMR $\left(\mathrm{C}_{6} \mathrm{D}_{6}\right): \delta 8.58(\mathrm{~d}, J=7.8 \mathrm{~Hz}, \mathrm{H}), 7.75(\mathrm{~d}, J=9.0 \mathrm{~Hz}, \mathrm{H}), 7.70(\mathrm{~d}, J=9.6 \mathrm{~Hz}, \mathrm{H}), 7.66(\mathrm{~d}, J=7.2 \mathrm{~Hz}$, $\mathrm{H}), 7.63(\mathrm{~d}, J=6.6 \mathrm{~Hz}, \mathrm{H}), 7.32(\mathrm{~m}, 4 \mathrm{H}), 7.18(\mathrm{~d}, J=8.4 \mathrm{~Hz}, \mathrm{H}), 6.99(\mathrm{~d}, J=7.8 \mathrm{~Hz}, \mathrm{H}), 6.39(\mathrm{~s}, \mathrm{H}, \mathrm{NH})$, $2.93(\mathrm{~m}, \mathrm{H}), 2.79(\mathrm{~m}, \mathrm{H}), 2.70(\mathrm{dt}, J=4.8 \mathrm{~Hz}, \mathrm{H}), 1.70(\mathrm{~m}, \mathrm{H}), 1.63(\mathrm{~m}, \mathrm{H}), 1.47(\mathrm{~m}, \mathrm{H}), 0.81(\mathrm{~d}, J=7.2 \mathrm{~Hz}$, $\left.3 \mathrm{H}, \mathrm{CH}\left(\mathrm{CH}_{3}\right)_{2}\right), 0.76\left(\mathrm{~d}, J=7.2 \mathrm{~Hz}, 3 \mathrm{H}, \mathrm{CH}\left(\mathrm{CH}_{3}\right)_{2}\right) \mathrm{ppm} .{ }^{13} \mathrm{C}\left\{{ }^{1} \mathrm{H}\right\} \mathrm{NMR}\left(\mathrm{C}_{6} \mathrm{D}_{6}\right): \delta 18.34,18.77,24.43$, 26.78, 32.52, 56.73, 112.78, 116.67, 122.62, 125.59, 126.10, 126.51, 126.61, 126.86, 128.14, 128.69, 129.03, $129.28,132.20,134.71,136.41,137.64,139.79,141.75,155.92 \mathrm{ppm} . \mathrm{m} / z$ calcd. $\left(\left[\mathrm{M}^{+}\right] \mathrm{C}_{25} \mathrm{H}_{24} \mathrm{~N}_{2}\right) 352.4800$. Found: 352.1942.

\subsection{Preparation of $\mathbf{1 5}$}

Complex 15 was prepared by the same procedure and experimental conditions as those employed for 10, using $13(0.366 \mathrm{~g}, 1.00 \mathrm{mmol})$. The product was sparingly soluble in hexane; therefore, it was extracted with toluene $(50 \mathrm{~mL})$. The trituration in hexane produced a dark brown powder $(0.259 \mathrm{~g}$, $45 \%) .{ }^{1} \mathrm{H}$ NMR $\left(\mathrm{C}_{6} \mathrm{D}_{6}\right): \delta 8.65(\mathrm{~d}, J=7.2 \mathrm{~Hz}, \mathrm{H}), 8.52(\mathrm{~d}, J=8.4 \mathrm{~Hz}, \mathrm{H}), 7.95(\mathrm{~d}, J=9.0 \mathrm{~Hz}, \mathrm{H}), 7.87(\mathrm{~d}$, $J=7.8 \mathrm{~Hz}, \mathrm{H}), 7.77(\mathrm{~d}, J=8.4 \mathrm{~Hz}, \mathrm{H}), 7.64(\mathrm{~d}, J=9.0 \mathrm{~Hz}, \mathrm{H}), 7.41(\mathrm{t}, J=7.8 \mathrm{~Hz}, \mathrm{H}), 7.32(\mathrm{t}, J=7.8 \mathrm{~Hz}, \mathrm{H})$, $7.18(\mathrm{~d}, J=8.4 \mathrm{~Hz}, \mathrm{H}), 6.81(\mathrm{~d}, J=8.4 \mathrm{~Hz}, \mathrm{H}), 4.33(\mathrm{~m}, \mathrm{H}), 2.88(\mathrm{~m}, \mathrm{H}), 2.57(\mathrm{dt}, J=3.6 \mathrm{~Hz}, \mathrm{H}), 2.11(\mathrm{~m}$, $\mathrm{H}), 1.92(\mathrm{~m}, \mathrm{H}), 1.79(\mathrm{~m}, \mathrm{H}), 1.38(\mathrm{~m}, 4 \mathrm{H}), 0.96\left(\mathrm{t}, J=6.6 \mathrm{~Hz}, 3 \mathrm{H},\left(\mathrm{CH}_{2}\right)_{3} \mathrm{CH}_{3}\right), 0.83\left(\mathrm{~s}, 3 \mathrm{H}, \mathrm{HfCH}_{3}\right), 0.82$ $\left(\mathrm{s}, 3 \mathrm{H}, \mathrm{HfCH}_{3}\right)$ ppm. ${ }^{13} \mathrm{C}\left\{{ }^{1} \mathrm{H}\right\} \mathrm{NMR}\left(\mathrm{C}_{6} \mathrm{D}_{6}\right): \delta 14.46,23.20,24.70,28.82,35.47,53.35,62.87,65.21,112.16$, $119.18,119.65,124.47,125.46,126.69,127.04,129.64,130.00,130.22,131.27,133.32,135.59,140.81,141.69$, 144.07, 149.83, 164.16, 208.15 ppm. Anal. Calcd. $\left(\mathrm{C}_{28} \mathrm{H}_{30} \mathrm{HfN}_{2}\right): \mathrm{C}, 58.69 ; \mathrm{H}, 5.28 ; \mathrm{N}, 4.89 \%$. Found: $\mathrm{C}$, $58.79 ; \mathrm{H}, 5.21 ; \mathrm{N}, 4.87 \%$.

\subsection{Preparation of 16}

Complex 16 was prepared by the same procedure and experimental conditions as those employed for 10, using $14(0.303 \mathrm{~g}, 0.859 \mathrm{mmol})$. The product was sparingly soluble in hexane; therefore, it was extracted with toluene $(50 \mathrm{~mL})$. The trituration in hexane produced a dark brown powder $(0.226 \mathrm{~g}$, $47 \%) .{ }^{1} \mathrm{H}$ NMR $\left(\mathrm{C}_{6} \mathrm{D}_{6}\right): \delta 8.66(\mathrm{~d}, J=7.8 \mathrm{~Hz}, \mathrm{H}), 8.50(\mathrm{~d}, J=7.8 \mathrm{~Hz}, \mathrm{H}), 7.92(\mathrm{~d}, J=9.0 \mathrm{~Hz}, \mathrm{H}), 7.83(\mathrm{~d}$, $J=7.2 \mathrm{~Hz}, \mathrm{H}), 7.76(\mathrm{~d}, J=8.4 \mathrm{~Hz}, \mathrm{H}), 7.62(\mathrm{~d}, J=7.8 \mathrm{~Hz}, \mathrm{H}), 7.40(\mathrm{td}, J=7.2 \mathrm{~Hz}, \mathrm{H}), 7.32(\mathrm{~m}, \mathrm{H}), 7.14$ $(\mathrm{d}, J=7.8 \mathrm{~Hz}, \mathrm{H}), 6.77(\mathrm{~d}, J=7.2 \mathrm{~Hz}, \mathrm{H}), 4.02(\mathrm{~m}, \mathrm{H}), 2.80(\mathrm{~m}, \mathrm{H}), 2.62(\mathrm{dt}, J=6.0 \mathrm{~Hz}, \mathrm{H}), 2.55(\mathrm{~m}, \mathrm{H})$, $1.88(\mathrm{~m}, \mathrm{H}), 1.72(\mathrm{~m}, \mathrm{H}), 1.09$ and $1.04\left(\mathrm{~d}, J=6.6 \mathrm{~Hz}, 6 \mathrm{H}, \mathrm{CH}\left(\mathrm{CH}_{3}\right)_{2}\right), 0.82\left(\mathrm{~s}, 3 \mathrm{H}, \mathrm{HfCH}_{3}\right), 0.81(\mathrm{~s}, 3 \mathrm{H}$, $\left.\mathrm{HfCH}_{3}\right)$ ppm. ${ }^{13} \mathrm{C}\left\{{ }^{1} \mathrm{H}\right\} \mathrm{NMR}\left(\mathrm{C}_{6} \mathrm{D}_{6}\right): \delta 18.55,21.28,23.07,25.44,32.58,60.98,63.06,66.88,112.37,119.64$, $120.21,124.55,125.48,126.81,126.97,129.31,129.97,130.26,131.25,133.82,135.51,140.97,141.44,143.94$, 
150.14, 164.58, 209.13 ppm. Anal. Calcd. $\left(\mathrm{C}_{27} \mathrm{H}_{28} \mathrm{HfN}_{2}\right)$ : C, 58.01; H, 5.05; N, 5.01\%. Found: C, 57.91; $\mathrm{H}, 5.01 ; \mathrm{N}, 5.11 \%$.

\subsection{Preparation of Anhydrous $\left[\left(\mathrm{C}_{18} \mathrm{H}_{37}\right)_{2} \mathrm{~N}(\mathrm{H}) \mathrm{Me}\right]^{+}\left[B\left(\mathrm{C}_{6} \mathrm{~F}_{5}\right)_{4}\right]^{-}$}

$\left[\left(\mathrm{C}_{18} \mathrm{H}_{37}\right)_{2} \mathrm{~N}(\mathrm{H}) \mathrm{Me}\right]^{+}\left[\mathrm{B}\left(\mathrm{C}_{6} \mathrm{~F}_{5}\right)_{4}\right]^{-}$, which was prepared according to the method reported in patent, contained water, [36] which caused some problems in the activation reactions. The water contained in $\left[\left(\mathrm{C}_{18} \mathrm{H}_{37}\right)_{2} \mathrm{~N}(\mathrm{H}) \mathrm{Me}\right]^{+}\left[\mathrm{B}\left(\mathrm{C}_{6} \mathrm{~F}_{5}\right)_{4}\right]^{-}$was not removed by the conventional ways (i.e., evacuation at $60^{\circ} \mathrm{C}$, refluxing with the Dean-Stark apparatus after dissolving in toluene, or treatment with molecular sieves in methylcyclohexane). The ${ }^{19} \mathrm{~F}$ NMR spectrum indicated that the $\mathrm{K}^{+}\left[\mathrm{B}\left(\mathrm{C}_{6} \mathrm{~F}_{5}\right)_{4}\right]^{-}$that was purchased from Alfa Aesar, contained $10 \mathrm{~mol} \%$ impurity. Therefore, excess $\mathrm{K}^{+}\left[\mathrm{B}\left(\mathrm{C}_{6} \mathrm{~F}_{5}\right)_{4}\right]^{-}(0.633 \mathrm{~g}$, $0.881 \mathrm{mmol}$, based on the assumption that it is pure) was reacted with $\left[\left(\mathrm{C}_{18} \mathrm{H}_{37}\right)_{2} \mathrm{~N}(\mathrm{H}) \mathrm{Me}\right]^{+}[\mathrm{Cl}]^{-}$ $(0.404 \mathrm{~g}, 0.705 \mathrm{mmol})$ in toluene (anhydrous, $10 \mathrm{~mL})$, for $1 \mathrm{~h}$, at room temperature, inside a glove box. After filtration over Celite, the solvent was removed using a vacuum line. The residue was dissolved in methylcyclohexane $(4 \mathrm{~mL})$ and filtered again over Celite. The removal of the solvent produced a yellow oily compound, which was used without further purification $(0.797 \mathrm{~g}, 93 \%)$. In the ${ }^{1} \mathrm{H}$ NMR spectrum of the water-containing $\left[\left(\mathrm{C}_{18} \mathrm{H}_{37}\right)_{2} \mathrm{~N}(\mathrm{H}) \mathrm{Me}\right]^{+}\left[\mathrm{B}\left(\mathrm{C}_{6} \mathrm{~F}_{5}\right)_{4}\right]^{-}$, prepared according to the patent method, $\mathrm{NCH}_{2}$ protons were observed as a single broad signal around $1.89 \mathrm{ppm}$ (Figure S28). In contrast, in

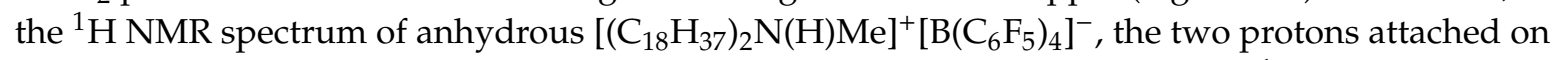
the $\alpha$-carbon $\left(\mathrm{NCH}_{2}\right)$ are separately observed at 1.97 and $1.80 \mathrm{ppm}$ (Figure $\left.\mathrm{S} 27\right) .{ }^{1} \mathrm{H}$ NMR $\left(\mathrm{C}_{6} \mathrm{D}_{6}\right): \delta$ $3.15(\mathrm{br}, \mathrm{H}, \mathrm{NH}), 1.97\left(\mathrm{~m}, 2 \mathrm{H}, \mathrm{NCH}_{2}\right), 1.80\left(\mathrm{~m}, \mathrm{H}, \mathrm{NCH}_{2}\right), 1.51\left(\mathrm{~d}, J=6.0 \mathrm{~Hz}, 3 \mathrm{H}, \mathrm{NCH}_{3}\right), 1.45-1.29(\mathrm{~m}$, $48 \mathrm{H}$ ), 1.26 (quintet, $J=7.2 \mathrm{~Hz}, 4 \mathrm{H}$ ), 1.13 (quintet, $J=7.2 \mathrm{~Hz}, 4 \mathrm{H}), 0.94(\mathrm{t}, J=7.8 \mathrm{~Hz}, 6 \mathrm{H}$ ), 0.88 (quintet, $J=7.8 \mathrm{~Hz}, 4 \mathrm{H}), 0.81(\mathrm{~m}, 4 \mathrm{H}) \mathrm{ppm} .{ }^{19} \mathrm{~F} \mathrm{NMR}\left(\mathrm{C}_{6} \mathrm{D}_{6}\right): \delta-132.09,-161.75,-165.98$.

\subsection{A Typical Polymerization (Entry 8 in Table 1)}

A bomb reactor $(125 \mathrm{~mL})$ was evacuated at $60{ }^{\circ} \mathrm{C}$ for $1 \mathrm{~h}$. After charging with ethylene gas at atmospheric pressure, a solution of $\mathrm{Me}_{3} \mathrm{Al}(28.8 \mathrm{mg}, 200 \mu \mathrm{mol}-\mathrm{Al})$ in methylcyclohexane (15.5 g) was added to the reactor. The mixture was stirred for $1 \mathrm{~h}$ at $100{ }^{\circ} \mathrm{C}$ using a mantle, and the solution was subsequently removed using a cannula. The reactor was evacuated once more to remove any residual solvent and was re-charged with ethylene gas at atmospheric pressure. This procedure was performed to clean up any catalyst poisons. The reactor was charged with methylcyclohexane $(15.5 \mathrm{~g})$, which contains MMAO (AkzoNobel, $6.7 \mathrm{wt} \%-\mathrm{Al}$ in heptane, $20 \mathrm{mg}, 50 \mu \mathrm{mol}-\mathrm{Al}$ ) and the temperature was set to $80{ }^{\circ} \mathrm{C}$. A solution of (octyl $)_{2} \mathrm{Zn}(100 \mu \mathrm{mol})$ in methylcyclohexane $(10.0 \mathrm{~g})$ was charged. Subsequently, the methylcyclohexane solution $(0.30 \mathrm{~g})$ containing the catalyst $12(2.0 \mu \mathrm{mol}-\mathrm{Hf})$ that was activated with $\left[\left(\mathrm{C}_{18} \mathrm{H}_{37}\right)_{2} \mathrm{~N}(\mathrm{H}) \mathrm{Me}^{+}\left[\mathrm{B}\left(\mathrm{C}_{6} \mathrm{~F}_{5}\right)_{4}\right]^{-}(1.0 \mathrm{eq})\right.$ in benzene, was injected. Ethylene/propylene mixed gas (10 bar/10 bar, total 20 bar) was charged from a tank into the reactor at 20 bar, and the polymerization was performed for $50 \mathrm{~min}$. The temperature was controlled at $80-90^{\circ} \mathrm{C}$. The remaining ethylene/propylene mixed gas was vented off and the reactor was cooled to $75^{\circ} \mathrm{C}$. The generated polymer was collected and dried in a vacuum oven at $160^{\circ} \mathrm{C}$ overnight to obtain $6.3 \mathrm{~g}$ of the polymer.

\subsection{X-ray Crystallography}

The reflection data for $\mathbf{1}, \mathbf{3}, \mathbf{6}, \mathbf{1 0}, \mathbf{1 1}$, and $\mathbf{1 2}$ were collected on a Bruker APEX II CCD area diffractometer (Billerica, MA, USA), using graphite-monochromated Mo K- $\alpha$ radiation $(\lambda=0.7107 \AA$ ). Specimens of suitable quality and sizes were selected, mounted, and centered in the X-ray beam using a video camera. The hemisphere of the reflection data was collected as $\varphi$ and $\omega$ scan frames at $0.5^{\circ} /$ frame and an exposure time of $10 \mathrm{~s} /$ frame. The cell parameters were determined and refined by the SMART program. Data reduction was performed using the SAINT software (Madison, WI, US). The data were corrected for Lorentz and polarization effects. An empirical absorption correction was applied using the SADABS program. The structures of the compounds were determined by direct methods and refined by the full matrix least-squares methods, using the SHELXTL program package with anisotropic thermal parameters for all non-hydrogen atoms. CCDC 1918314-1918319 
contain the supplementary crystallographic data for this paper. These data can be obtained free of charge via http://www.ccdc.cam.ac.uk/conts/retrieving.html (or from the CCDC 12 Union Road, Cambridge CB2 1EZ, UK; Fax: +44 1223 336033; E-mail: deposit@ccdc.cam.ac.uk). Crystallographic data for 1 (CCDC\# 1918314): $\mathrm{C}_{20} \mathrm{H}_{27} \mathrm{HfN}, M=459.91$, monoclinic, $a=7.7840(2), b=31.3344(6)$, $c=7.7852(2) \AA, \beta=104.9576(15)^{\circ}, V=1834.52(8) \AA^{3}, T=100(2) K$, space group $P 2_{1} / n, Z=4,3395$ unique $(\mathrm{R}(\mathrm{int})=0.1398)$, which were used in all the calculations. The final $w R_{2}$ was $0.0831(I>2 \sigma(I))$. Data for 3 (CCDC\# 1918315): $\mathrm{C}_{21} \mathrm{H}_{25} \mathrm{HfNS}, M=501.97$, monoclinic, $a=10.7974(2), b=11.5868(2), c=15.5040(3)$ $\AA, \beta=93.1287(9)^{\circ}, V=1936.77(6) \AA^{3}, T=296 \mathrm{~K}$, space group $P 2_{1} / c, Z=4,3563$ unique (R(int) $\left.=0.0350\right)$, which were used in all calculations. The final $w R_{2}$ was $0.0349(I>2 \sigma(I))$. Data for 6 (CCDC\# 1918316): $\mathrm{C}_{25} \mathrm{H}_{25} \mathrm{HfN}, M=517.95$, monoclinic, $a=8.7930(2), b=14.7370(3), c=15.7492(3) \AA, \beta=92.1487(10)^{\circ}$, $V=2039.38(7) \AA^{3}, T=100(2) \mathrm{K}$, space group $P 2_{1} / c, Z=4,4256$ unique $(\mathrm{R}(\mathrm{int})=0.0327)$, which were used in all the calculations. The final $w R_{2}$ was $0.0496(I>2 \sigma(I))$. Data for $10(C C D C \# 1918317): C_{20} \mathrm{H}_{18} \mathrm{HfN}_{2}$, $M=464.85$, orthorhombic, $a=12.5672(8), b=7.8737(5), c=34.8321(19) \AA, V=3446.6(4) \AA^{3}, T=100(2) \mathrm{K}$, space group $P b c a, Z=8,1002$ unique $(\mathrm{R}(\mathrm{int})=0.2063)$, which were used in all the calculations. The final $w R_{2}$ was $0.0807(I>2 \sigma(I))$. Data for 11 (CCDC\# 1918319): $\mathrm{C}_{24.70} \mathrm{H}_{28.60} \mathrm{HfN}_{2}, M=531.98$, monoclinic, $a=16.1724(3), b=16.1772(3), c=22.6003(4) \AA, \beta=107.9164(12)^{\circ}, V=5626.05(18) \AA^{3}, T=100(2) \mathrm{K}$, space group $P 2_{1}, Z=10,20485$ unique $(R($ int $)=0.1342)$, which were used in all the calculations. The final $w R_{2}$ was $0.1062(I>2 \sigma(I))$. Data for 12 (CCDC\# 1918318): $\mathrm{C}_{23} \mathrm{H}_{26.20} \mathrm{HfN}_{2}, M=509.15$, orthorhombic, $a=16.2081(7), b=24.608(1), c=27.4705(12) \AA, V=10956.6(8) \AA^{3}, T=100(2) \mathrm{K}$, space group $P 2{ }_{1}{ }_{2}{ }_{1} 2_{1}$, $Z=20,7082$ unique $(R($ int $)=0.1888)$, which were used in all calculations. The final $w R_{2}$ was 0.1383 $(I>2 \sigma(I))$.

\section{Results and Discussion}

\subsection{Preparation of Hf Complexes}

A series of Ti- and Zr-based half-metallocene complexes [ortho- $\left.\mathrm{C}_{6} \mathrm{H}_{4}(\mathrm{~L})(\mathrm{NR})\right] \mathrm{MMe}_{2}(\mathrm{M}=\mathrm{Ti}$ or $\mathrm{Zr})$ were prepared for ethylene $/ \alpha$-olefin copolymerizations. It was found that the Ti-complexes with $\mathrm{L}$ being tetramethylcyclopentadienyl or thiophene-fused dimethylcyclopentadienyl and $\mathrm{R}$ being linked to ortho-phenylene bridge exhibited excellent catalytic performance $[33,34,37,38]$. Titanium complexes were prepared on a large scale simply by treating the ligand precursor successively with 4 equiv $\mathrm{MeMgCl}$ and $\mathrm{TiCl}_{4}$.(DME) (DME = dimethoxyethane). Attempts to synthesize the Hf-analogues by the same procedure (i.e., treatment of the ligand precursor successively with four equiv $\mathrm{MeMgCl}$ and $\mathrm{HfCl}_{4}$-(THF) $)_{2}$ were unsuccessful [39]. The syntheses of Ti- and $\mathrm{Zr}$-based half-metallocene complexes have been widely reported. However, the syntheses of $\mathrm{Hf}$-analogues are seldom reported [40]. [ $\left.\mathrm{Me}_{2} \mathrm{Si}\left(\eta^{5}-\mathrm{Me}_{4} \mathrm{C}_{5}\right)\left(\mathrm{N}^{t} \mathrm{Bu}\right)\right] \mathrm{HfCl}_{2}$ was obtained in a rather low yield (38\%) by reacting $\left[\mathrm{Me}_{2} \mathrm{Si}\left(\eta^{5}-\mathrm{Me}_{4} \mathrm{C}_{5}\right)\left(\mathrm{N}^{t} \mathrm{Bu}\right)\right] \mathrm{Li}_{2}$ with $\mathrm{HfCl}_{4} \cdot(\mathrm{THF})_{2}$, whereas the corresponding reaction with $\mathrm{ZrCl}_{4} \cdot(\mathrm{THF})_{2}$ afforded the desired complex [ $\left.\mathrm{Me}_{2} \mathrm{Si}\left(\eta^{5}-\mathrm{Me}_{4} \mathrm{C}_{5}\right)\left(\mathrm{N}^{t} \mathrm{Bu}\right)\right] \mathrm{ZrCl}_{2}$ in high yield (74\%) [41,42]. Eventually, we found that some commercial sources of $\mathrm{HfCl}_{4}$ contained water, which was the cause of the failure. The use of sublimed-grade of $\mathrm{HfCl}_{4}$ instead of its water-containing counterpart, and $\mathrm{MeMgBr}$ instead of $\mathrm{MeMgCl}$, cleanly afforded the desired half-metallocene Hf complexes in good yield (84\%) (Scheme 2). Along with tetrahydroquinoline- and tetrahydroquinaldine-linked tetramethylcyclopentadienyl $\mathrm{HfMe}_{2}$ complexes (1 and 2), and tetrahydroquinoline- and tetrahydroquinaldine-linked thiophene-fused dimethylcyclopentadienyl $\mathrm{HfMe}_{2}$ complexes ( 3 and 4 ), of which $\mathrm{Ti}$ analogues were reported to show excellent performance, fluorenyl congeners ( 5 and 6 ) were also prepared because the excellent performance of $\left[\mathrm{Me}_{2} \mathrm{Si}\left(\eta^{5}\right.\right.$-fluorenyl)(NR)]TiMe 2 has also been reported [43]. The ${ }^{1} \mathrm{H}$ NMR and ${ }^{13} \mathrm{C}$ NMR spectra were in agreement with the expected structures (Figures S1-S6) and the structures of 1, 3, and 6 were confirmed by X-ray crystallography. 

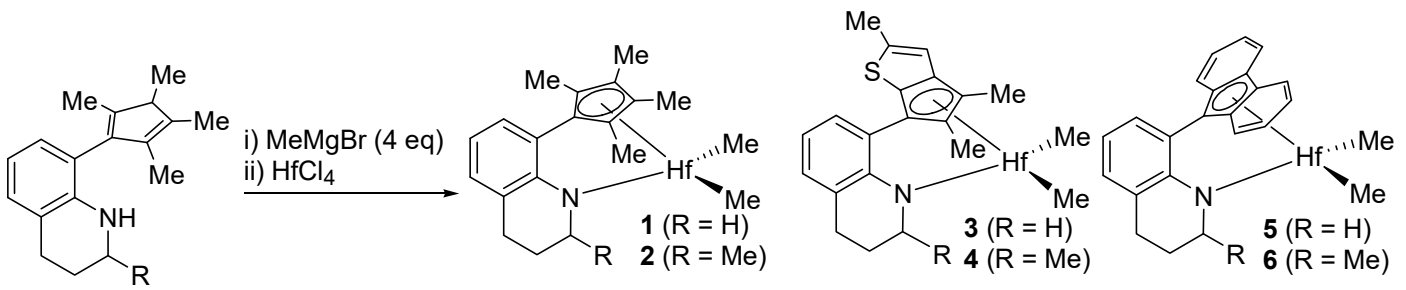

Scheme 2. Synthesis of Hf-based half-metallocene complexes.

Pyridylamido-Hf complex III is a [ $\left.\mathrm{N}^{\text {amido }}, \mathrm{N}^{\text {pyridine }}, \mathrm{C}^{\text {aryl }}\right] \mathrm{HfMe}_{2}$-type complex, which contains characteristic Hf-C(aryl) bonds [44]. Through olefin insertion into the Hf-C(aryl) bond at the initial stage of polymerization, the ligand structure was modified and accordingly, the coordination geometry changed significantly $[9,45]$. Polymer chains are grown from the active species with a modified ligand structure. Namely, the Hf-C(aryl) bond plays a critical role in the high performance; we attempted, in this work, to prepare similar [ $\left.\mathrm{N}^{\text {amido }}, \mathrm{N}, \mathrm{C}^{\text {aryl }}\right] \mathrm{HfMe}_{2}$-type complexes containing the $\mathrm{Hf}-\mathrm{C}$ (aryl) bond, with tetrahydrophenanthroline framework (Scheme 3). 9-Phenyl-1,2,3,4-tetrahydro [1,10] phenanthroline (7) and 2-butyl-9-phenyl-1,2,3,4-tetrahydro[1,10]phenanthroline (8) were known compounds and 2-isopropyl-9-phenyl-1,2,3,4-tetrahydro[1,10]phenanthroline (9) was synthesized by the modification of the reported method and conditions (Scheme 3a) [35]. The treatment of 7-9 with $\mathrm{HfMe}_{4}$, generated in situ by the reaction of four equiv $\mathrm{MeMgBr}$ and $\mathrm{HfCl}_{4}$, afforded the targeted $\left[\mathrm{N}^{\text {amido }}, \mathrm{N}, \mathrm{C}^{\text {aryl }}\right] \mathrm{HfMe}_{2}$-type complexes, which contain a Hf-C(aryl) bond [46]. $\mathrm{HfMe}_{4}$ is unstable; therefore, it should be generated and reacted in situ at a low temperature $\left(-35\right.$ to $\left.-40{ }^{\circ} \mathrm{C}\right)$. The Hf-C(aryl) bond formation was evident from the result of the ${ }^{1} \mathrm{H}$ NMR spectrum analysis (Figure 1a). The ortho-metalated phenylene $\left(\mathrm{N}=\mathrm{C}-\mathrm{C}_{6} \mathrm{H}_{4}-\mathrm{Hf}\right)$ signals were clearly observed at $8.50(\mathrm{~d}), 7.84(\mathrm{t}), 7.46(\mathrm{t})$, and $7.26(\mathrm{~d})$ ppm with integration value ratios of 1:1:1:1 in the ${ }^{1} \mathrm{H}$ NMR spectrum, whereas phenyl $\left(-\mathrm{C}_{6} \mathrm{H}_{5}\right)$ signals were observed at $8.18(\mathrm{~d}), 7.52(\mathrm{t})$, and $7.45(\mathrm{t}) \mathrm{ppm}$ with integration value ratios of 2:2:1 in those of ligand precursors 7-9. The structures of 10-12 were unambiguously confirmed by X-ray crystallography.

(a)

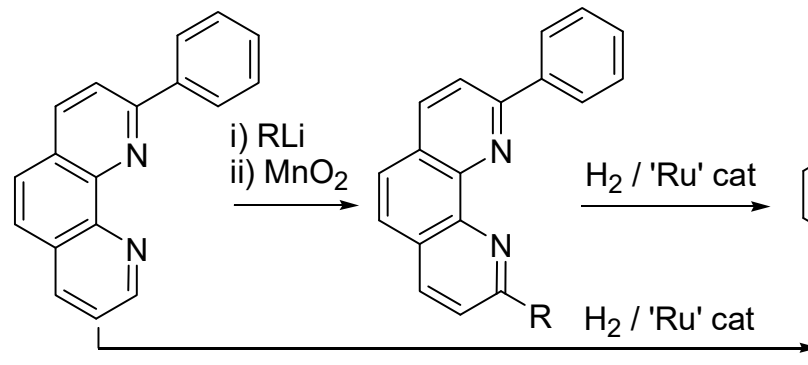<smiles>[R]C1CCc2ccc3ccc(-c4ccccc4)nc3c2N1</smiles>
$7(\mathrm{R}=\mathrm{H})$

$8(\mathrm{R}=\mathrm{nBu})$<smiles>[R]C1CCc2ccc3ccc(-c4ccccc4C(C)(C)C)n(c2-3)N1C</smiles>

$10(\mathrm{R}=\mathrm{H})$

$11(\mathrm{R}=\mathrm{nBu})$$$
12(\mathrm{R}=\mathrm{iPr})
$$

(b)<smiles>C1CCCCC1</smiles><smiles>[R]C1C=Cc2ccc3ccc(C4=CCCc5ccccc54)nc3c2N1</smiles>

\section{$\mathrm{H}_{2} / \mathrm{Pd} / \mathrm{C}$}<smiles>[R]C1CCc2ccc3ccc(-c4cccc5ccccc45)nc3c2N1</smiles>

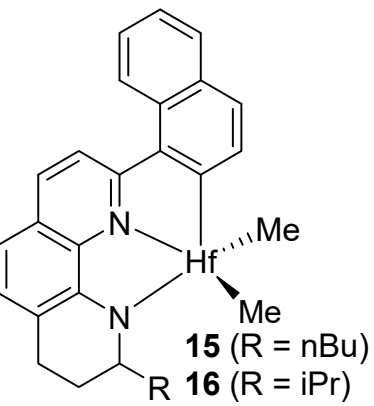

Scheme 3. Synthesis of $\left[\mathrm{N}^{\text {amido }}, \mathrm{N}, \mathrm{C}^{\text {aryl }}\right] \mathrm{HfMe}_{2}$-type post-metallocene complexes bearing phenyl moiety (a) and naphthyl moiety (b). 
(a)

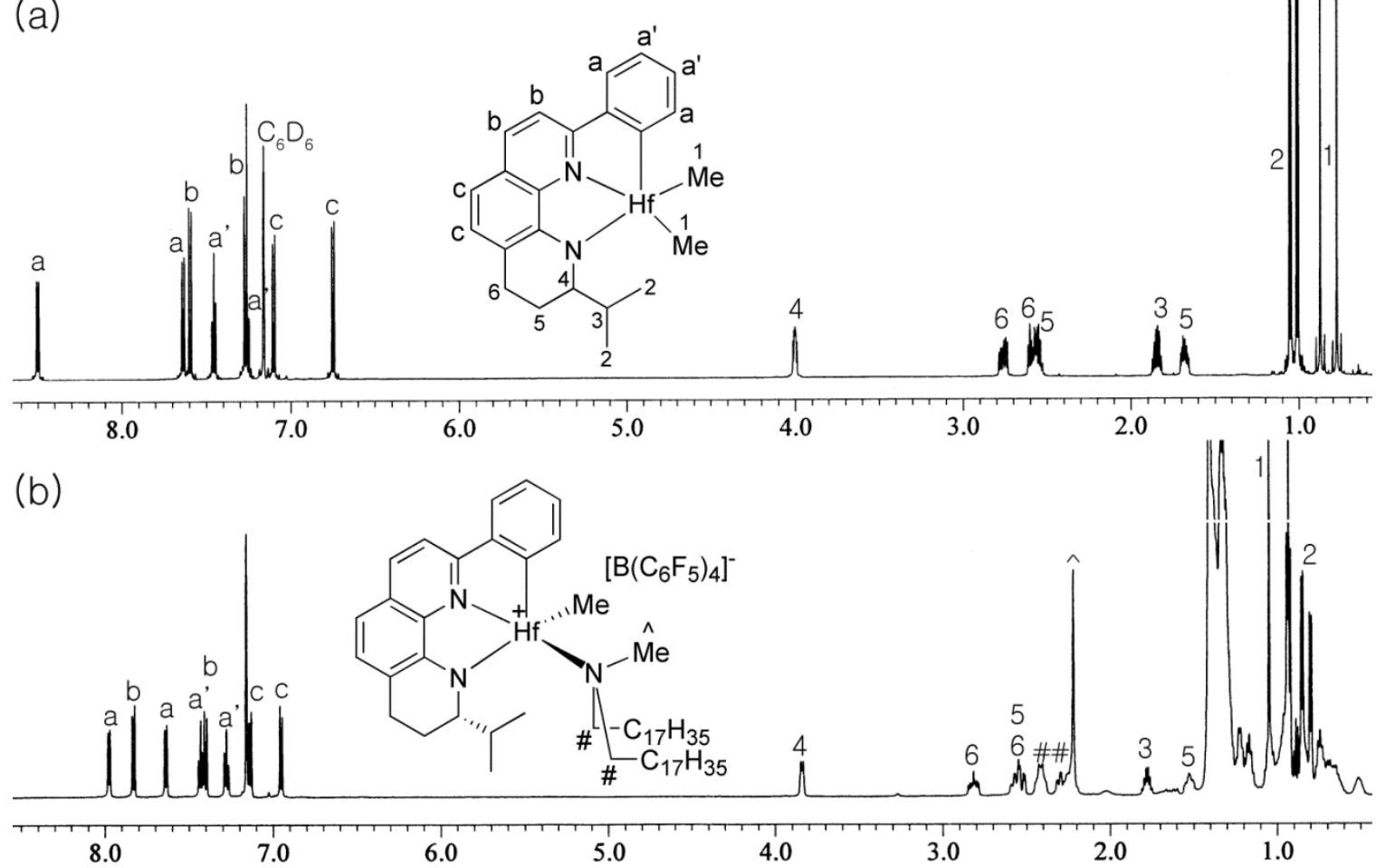

Figure 1. ${ }^{1} \mathrm{H}$ NMR spectra of $\mathbf{1 2}$ (a) and its activated complex (b).

Analogues ligand precursors containing a naphthyl substituent instead of phenyl (13 and $\mathbf{1 4}$ in Scheme 3b) were not synthesized according to the same synthetic scheme, because naphthyl group was also involved in the hydrogenation process. $\mathbf{1 3}$ and $\mathbf{1 4}$ were successfully synthesized through the selective hydrogenation of the intermediates that were captured from the reaction of 2-naphthylphenanthroline with RLi. To avoid the hydrogenation of the naphthyl group, the Pd/C catalyst was used instead of the Ru-complex and $\mathrm{H}_{2}$ pressure was lowered to 5 bar. From 13 and 14, the targeted complexes (15 and 16) were successfully prepared by the treatment of the in situ generated $\mathrm{HfMe}_{4}$. The ${ }^{1} \mathrm{H}$ and ${ }^{13} \mathrm{C}$ NMR spectra were in agreement with the expected structure (Figures S13 and S14).

\subsection{X-ray Crystallographic Studies}

The molecular structures of the half-metallocene Hf complexes of 1, 3, and $\mathbf{6}$ were confirmed by X-ray crystallography (Figure 2). The sum of the bond angles around the $\mathrm{N}$ atom is in all cases $360^{\circ}$, indicating that the $\mathrm{N}$ atoms adopt a sp ${ }^{2}$-hybridization for the $\pi$-donation from $\mathrm{N}$ to the $\mathrm{Hf}$ atom. The Hf-N distances are in the order of $\mathbf{1}>\mathbf{3}>\mathbf{6}$ (2.150(2), 2.038(2), and 2.022(3) $\mathrm{A}$, respectively) and these are substantially longer than the Ti-N distances observed for the corresponding Ti complexes (1.929(2), 1.936(3), and 1.921(2) $\AA$, respectively) [33,34]. The $C_{5}$ (centroid)-Hf distances are reversely in the order of $\mathbf{6}>\mathbf{3}>\mathbf{1}$, and the observed distance for $\mathbf{1}(2.01 \AA)$, is similar to the $C_{5}$ (centroid)-Ti distance observed for the corresponding Ti complex (2.02 $\AA$ ), while those for 3 and $\mathbf{6}(2.17$ and $2.22 \AA$ ) are longer than the $\mathrm{C}_{5}$ (centroid)-Ti distances observed for the corresponding Ti complexes (2.03 and $2.07 \AA$ ). The $\mathrm{C}_{5}$ (centroid)-Hf-N angles $\left(102.2^{\circ}, 102.1^{\circ}\right.$, and $102.4^{\circ}$ for $\mathbf{1}, 3$, and 6 , respectively) are substantially more acute than the $\mathrm{C}_{5}$ (centroid)-Ti-N angles observed for the corresponding Ti complexes $\left(106.1^{\circ}\right.$, $106.8^{\circ}$, and $106.9^{\circ}$ ). The $\mathrm{C}_{5}$ (centroid)-Ti-N angles have been used as a qualitative measure for the constrained geometry. The more acute the angle, the more open the reaction site becomes. 
(a)

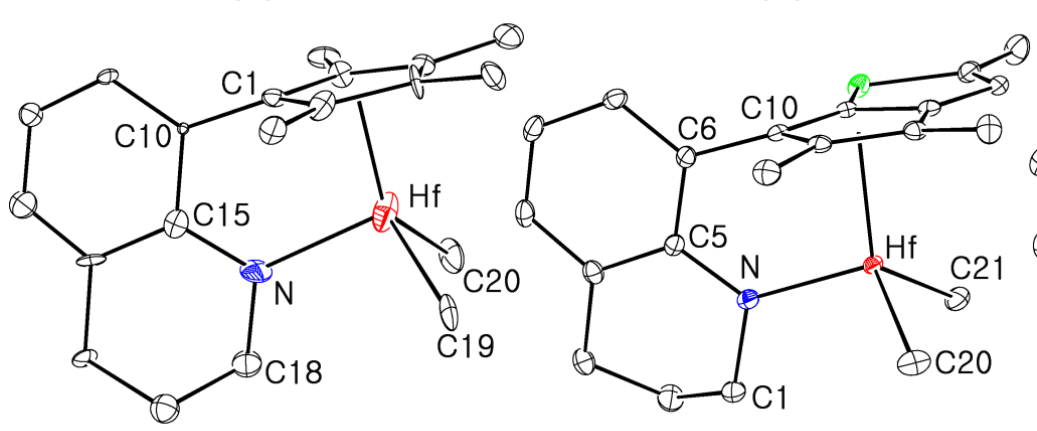

(C)

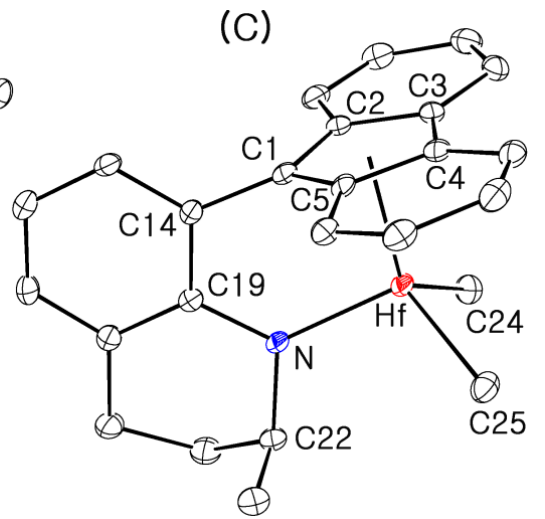

Figure 2. Thermal ellipsoid plot (30\% probability level) of $\mathbf{1}(\mathbf{a}), \mathbf{3}(\mathbf{b})$, and $\mathbf{6}(\mathbf{c})$. Hydrogen atoms are omitted for clarity. Selected bond distances $(\AA)$ and angles $\left({ }^{\circ}\right)$ in $\mathbf{1}(\mathbf{a})$ : Hf-N, 2.15(2): Hf-C(19), 2.20(2); Hf-C(20), 2.36(2); $\mathrm{C}_{5}$ (centroid)-Hf, 2.01; $\mathrm{C}_{5}$ (centroid)-Hf-N, 102.2; C(19)-Hf-C(20), 101.9(9); $\mathrm{C}_{5}$ (centroid)-C(1)-C(10), 170.1; C(1)- $\mathrm{C}_{5}$ (centroid)-Hf, 91.6; C(15)-N-Hf, 124.3(1); C(18)-N-Hf, 121.2(1); $\mathrm{C}(15)-\mathrm{N}-\mathrm{C}(18), 114.3(2)$. In 3 (b): Hf-N, 2.038(2): Hf-C(20), 2.217(3); Hf-C(21), 2.223(3); $\mathrm{C}_{5}$ (centroid)-Hf, 2.17; $\mathrm{C}_{5}$ (centroid)-Hf-N, 102.1; C(20)-Hf-C(21), 103.7(1); $\mathrm{C}_{5}$ (centroid)-C(10)-C(6), 171.1; $\mathrm{C}(10)-\mathrm{C}_{5}$ (centroid)-Hf, 88.2; C(1)-N-Hf, 117.0(2); C(5)-N-Hf, 126.9(2); C(1)-N-C(5), 116.1(2). In 6 (c): Hf-N, 2.022(3): Hf-C(24), 2.227(4); Hf-C(25), 2.510(3); $\mathrm{C}_{5}$ (centroid)-Hf, 2.22; C(1)-Hf, 2.451(3); C(2)-Hf, 2.505(3); C(5)-Hf, 2.510(3); C(3)-Hf, 2.540(3); C(4)-Hf, 2.541(3); $\mathrm{C}_{5}$ (centroid)-Hf-N, 102.4; C(24)-Hf-C(25), 103.7(1); $\mathrm{C}_{5}$ (centroid)-C(1)-C(14), 172.5; C(1)- $\mathrm{C}_{5}$ (centroid)-Hf, 86.7; C(19)-N-Hf, 126.5(2); C(22)-N-Hf, 118.8(2); C(19)-N-C(22), 114.5(2).

The molecular structures of the post-metallocene Hf complexes of 10, 11, and 12 were also confirmed by X-ray crystallography (Figure 3). Despite the unsatisfactory quality of the data, the molecular structures can be seen clearly. The coordination geometry can be defined as a distorted trigonal bipyramid; $\mathrm{N}$ (pyridine), $\mathrm{C}$ (methyl), $\mathrm{C}$ (methyl), and Hf atoms form a plane (sum of the bond angles around $\mathrm{Hf}$ atoms, $360^{\circ}$ ), while $\mathrm{C}$ (aryl) and $\mathrm{N}$ (amido) atoms distortedly occupy the axial sites (C(aryl)-Hf- $\mathrm{N}\left(\right.$ amido) angles, $140^{\circ}$ ). The sum of the bond angles around the $\mathrm{N}$ (amido) atoms in 10, 11 , and 12 are perfectly $360^{\circ}$ or close to $360^{\circ}\left(360^{\circ}, 360^{\circ}\right.$, and $357^{\circ}$, respectively), indicating that the $\mathrm{N}$ atoms adopt an $\mathrm{sp}^{2}$-hybridization for the $\pi$-donation from $\mathrm{N}$ to the $\mathrm{Hf}$ atom. All the atoms in the ligand framework except a $\mathrm{CH}_{2}$ (i.e., $\mathrm{N}-\mathrm{C}(\mathrm{R}) \mathrm{HCH}_{2}$ ) fragment, are situated nearly in a plane with the Hf atom, while the $\mathrm{C}$ (methyl)-Hf-C(methyl) plane perpendicularly bisects the plane of the ligand framework (the angle between the two planes, $87^{\circ}$ ). The butyl group in $\mathbf{1 1}$ is directed nearly perpendicularly from the plane of the ligand framework ( $\mathrm{Hf}-\mathrm{N}(4)-\mathrm{C}(42)-\mathrm{C}(43)$ dihedral angle, $\left.91^{\circ}\right)$, while the isopropyl group in 12 is askew form the plane (Hf-N(2)-C(18)-C(19) dihedral angle, $33^{\circ}$ ). The $\mathrm{Hf}-\mathrm{C}$ (methyl) distances are in the order of $\mathbf{1 2}(2.28(5), 2.27(5) \AA)>\mathbf{1 1}(2.20(2), 2.26(2) \AA)>\mathbf{1 0}(2.22(2), 2.22(2) \AA)$. 

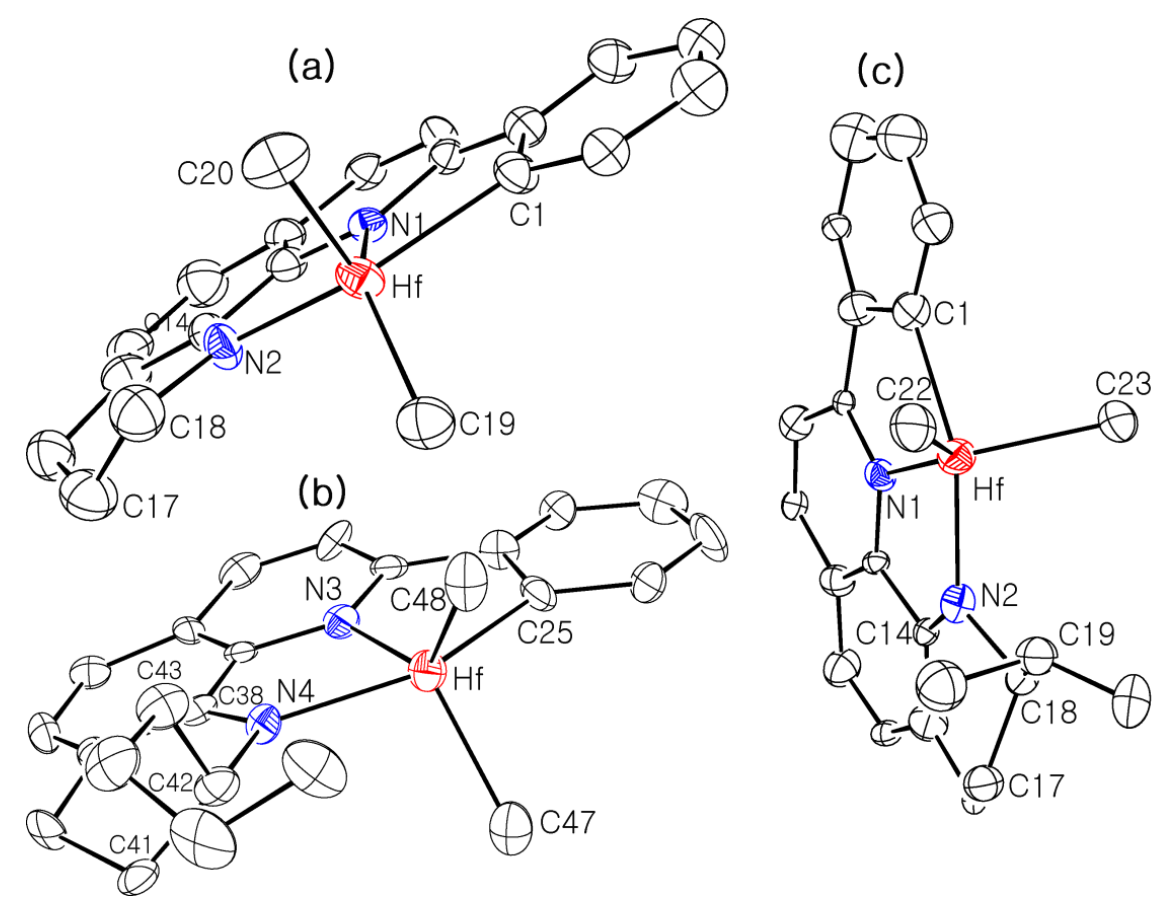

Figure 3. Thermal ellipsoid plot (30\% probability level) of $\mathbf{1 0}(\mathbf{a}), \mathbf{1 1}(\mathbf{b})$, and $\mathbf{1 2}(\mathbf{c})$. Hydrogen atoms are omitted for clarity. Selected bond distances $(\AA)$ and angles $\left(^{\circ}\right)$ in $\mathbf{1 0}(\mathbf{a})$ : Hf-N(1), 2.30(2); Hf-N(2), 2.11(2); Hf-C(1), 2.27(2); Hf-C(19), 2.22(2); Hf-C(20), 2.22(2); N(1)-Hf-C(19), 137.4(7); N(1)-Hf-C(20), 118.9(7); C(19)-Hf-C(20), 103.6(9); N(2)-Hf-C(1), 140.4(8); C(14)-N(2)-Hf, 124.2(1); C(18)-N(2)-Hf, 121.3(2); $\mathrm{C}(14)-\mathrm{N}(4)-\mathrm{C}(18), 114.5(2) ; \mathrm{Hf}-\mathrm{N}(2)-\mathrm{C}(18)-\mathrm{C}(17), 29.2$. In 11 (b): Hf-N(3), 2.30(2); Hf-N(4), 2.12(2); Hf-C(25), 2.25(2); Hf-C(47), 2.20(2); Hf-C(48), 2.26(2); N(3)-Hf-C(48), 133.5(8); N(3)-Hf-C(47), 124.2(8); C(47)-Hf-C(48), 102.1(1); N(4)-Hf-C(25), 139.7(9); C(38)-N(4)-Hf, 122.0(1); C(42)-N(4)-Hf, 121.2(2); C(38)-N(4)-C(42), 116.6(2); Hf-N(4)-C(42)-C(43), 91.1; Hf-N(4)-C(42)-C(41), 38.4. In 12 (c): Hf-N(1), 2.23(3); Hf-N(2), 2.14(3); Hf-C(1), 2.39(5); Hf-C(22), 2.28(5); Hf-C(23), 2.27(5); N(1)-Hf-C(22), 136.1(2); N(1)-Hf-C(23), 121.6(2); C(22)-Hf-C(23), 101.5(2); N(2)-Hf-C(1), 141.1(2); C(14)-N(2)-Hf, 122; $\mathrm{C}(18)-\mathrm{N}(2)-\mathrm{Hf}, 120 ; \mathrm{C}(14)-\mathrm{N}(2)-\mathrm{C}(18), 115 ; \mathrm{Hf}-\mathrm{N}(2)-\mathrm{C}(18)-\mathrm{C}(19), 33 ; \mathrm{Hf}-\mathrm{N}(2)-\mathrm{C}(18)-\mathrm{C}(17), 17$.

\subsection{Activation Reactions}

The activation reaction of the prototype pyridylamidohafnium complex III is rather tricky and complex $[47,48]$. The reaction with $\mathrm{B}\left(\mathrm{C}_{6} \mathrm{~F}_{5}\right)_{3}$ results in an activated complex; however, the generated complex was decomposed through a process involving $\mathrm{C}_{6} \mathrm{~F}_{5}$ transfers. The reaction with $\left[\mathrm{Ph}_{3} \mathrm{C}\right]^{+}\left[\mathrm{B}\left(\mathrm{C}_{6} \mathrm{~F}_{5}\right)_{4}\right]^{-}$may immediately afford the targeted ion-pair complex $\left\{\left[\mathrm{N}, \mathrm{N}, \mathrm{C}^{\text {naphthyl }}\right] \mathrm{HfMe}\right\}^{+}$ $\left[\mathrm{B}\left(\mathrm{C}_{6} \mathrm{~F}_{5}\right)_{4}\right]^{-}$, which also decomposes especially when exposed to sunlight or a polar solvent. The reaction with $\left.\left[\mathrm{PhN}(\mathrm{H}) \mathrm{Me}_{2}\right]^{+}\left[\mathrm{B}_{(} \mathrm{C}_{6} \mathrm{~F}_{5}\right)_{4}\right]^{-}$results in protonation on the $\mathrm{Hf}-\mathrm{C}^{\text {Naphthyl }}$ bond to generate a $\left.\{[\mathrm{N}, \mathrm{N}] \mathrm{HfMe}\}_{2}\right\}^{+}\left[\mathrm{B}\left(\mathrm{C}_{6} \mathrm{~F}_{5}\right)_{4}\right]^{-}$-type complex, which further reacts with the generated byproduct $\left(\mathrm{PhNMe}_{2}\right)$ to produce an undesired complex. The best activator was the aliphatic amine-based ammonium salt, $\left[\left(\mathrm{C}_{18} \mathrm{H}_{37}\right)_{2} \mathrm{~N}(\mathrm{H}) \mathrm{Me}\right]^{+}\left[\mathrm{B}\left(\mathrm{C}_{6} \mathrm{~F}_{5}\right)_{4}\right]^{-}$, which cleanly afforded the desired ion-pair complex, $\left\{\left[\mathrm{N}, \mathrm{N}, \mathrm{C}^{\text {naphthyl }}\right] \mathrm{HfMe}\right\}^{+}\left[\mathrm{B}\left(\mathrm{C}_{6} \mathrm{~F}_{5}\right)_{4}\right]^{-}$. The activated ion-pair complex is stable in benzene [18].

When the half-metallocene complex 1 was reacted with $\left[\left(\mathrm{C}_{18} \mathrm{H}_{37}\right)_{2} \mathrm{~N}(\mathrm{H}) \mathrm{Me}\right]^{+}\left[\mathrm{B}\left(\mathrm{C}_{6} \mathrm{~F}_{5}\right)_{4}\right]^{-}$in $\mathrm{C}_{6} \mathrm{D}_{6}$, a single set of signals was observed in the ${ }^{1} \mathrm{H}$ NMR spectrum (Figure S15), which was assigned to the desired ion-pair complex generated by the protonation on Hf-Me (Scheme 4a). The reaction was rather slow, requiring several hours, and the generated ion-pair complex was stable in $\mathrm{C}_{6} \mathrm{D}_{6} \cdot\left(\mathrm{CH}_{3}\right)_{4} \mathrm{C}_{5}$ signals were separately observed at $2.02,1.92,1.70$, and $1.58 \mathrm{ppm}$ as sharp singlets, which are indicative of the tight binding of $\left(\mathrm{C}_{18} \mathrm{H}_{37}\right)_{2} \mathrm{NMe}$ to a vacant site on the $\mathrm{Hf}$ center that was generated by the methide abstraction. At the structural point of amine tight binding, the Hf center becomes a chiral center; the two $\alpha$-methylene carbons and furthermore, the two protons attached on each $\alpha$-methylene 
carbon on $\left(\mathrm{C}_{18} \mathrm{H}_{37}\right)_{2} \mathrm{NMe}$, are inequivalent and $\mathrm{NCH}_{2}$ resonances were separately observed at 2.34, 2.26 , and $2.18 \mathrm{ppm}$. In the ${ }^{19} \mathrm{~F}$ NMR spectrum, signals assignable to ortho-, meta-, and para-fluorine of $-\mathrm{C}_{6} \mathrm{~F}_{5}$ were observed. The analyses of the ${ }^{1} \mathrm{H}$ NMR spectra of the complexes generated by the action of $\left[\left(\mathrm{C}_{18} \mathrm{H}_{37}\right)_{2} \mathrm{~N}(\mathrm{H}) \mathrm{Me}\right]^{+}\left[\mathrm{B}\left(\mathrm{C}_{6} \mathrm{~F}_{5}\right)_{4}\right]^{-}$to 2-6 indicated that the desired ion-pair complexes were cleanly generated (Figures S16-S20).

(a)

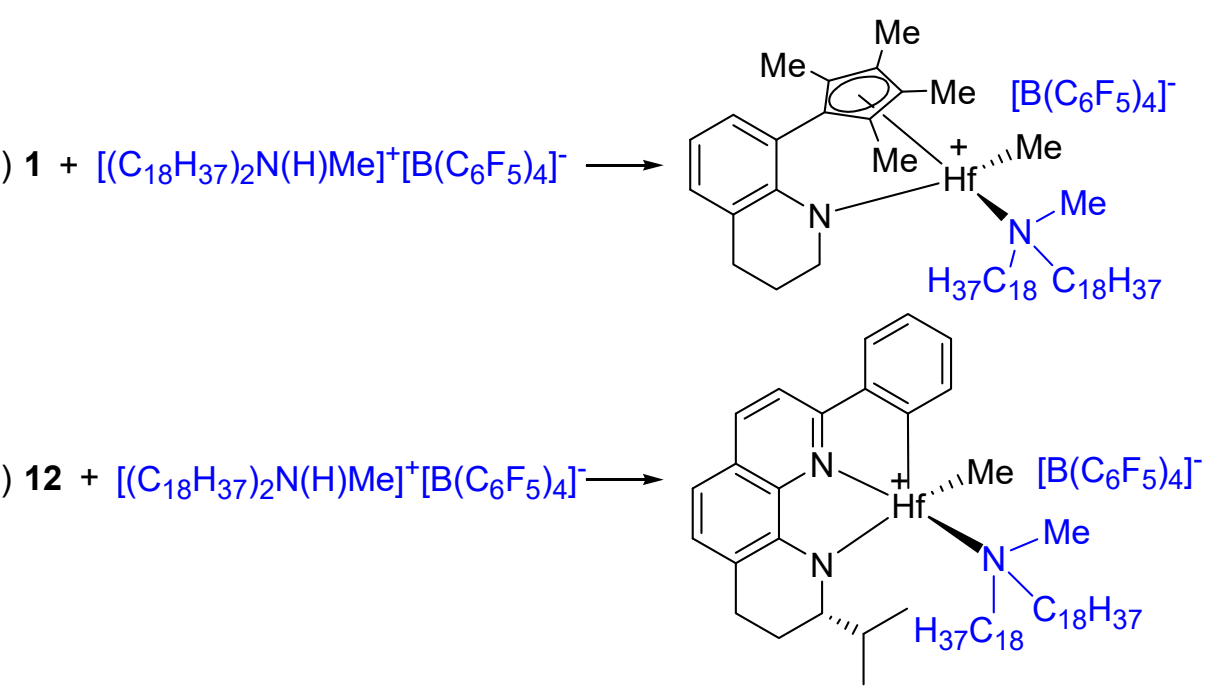

Scheme 4. Activation reaction of $\mathbf{1}(\mathbf{a})$ and $\mathbf{1 2}(\mathbf{b})$ with $\left[\left(\mathrm{C}_{18} \mathrm{H}_{37}\right)_{2} \mathrm{~N}(\mathrm{H}) \mathrm{Me}\right]^{+}\left[\mathrm{B}\left(\mathrm{C}_{6} \mathrm{~F}_{5}\right)_{4}\right]^{-}$.

When 12, bearing the isopropyl substituent, was reacted with $\left[\left(\mathrm{C}_{18} \mathrm{H}_{37}\right)_{2} \mathrm{~N}(\mathrm{H}) \mathrm{Me}\right]^{+}\left[\mathrm{B}\left(\mathrm{C}_{6} \mathrm{~F}_{5}\right)_{4}\right]^{-}$in $\mathrm{C}_{6} \mathrm{D}_{6}$, the desired ion-pair complex was immediately generated with the concomitant generation of methane (Scheme $4 \mathrm{~b}$ ). The generated complex was stable in $\mathrm{C}_{6} \mathrm{D}_{6}$ for several days. A single set of signals assignable to the desired ion-pair complex was observed in the ${ }^{1} \mathrm{H}$ NMR spectrum (Figure $1 \mathrm{~b}$ ). The Hf- $\mathrm{CH}_{3}$ signal was observed at $1.06 \mathrm{ppm}$ as a singlet. Amine $\left(\mathrm{C}_{18} \mathrm{H}_{37}\right)_{2} \mathrm{NMe}$ seems to bind to the Hf center rather loosely. The $\mathrm{NCH}_{2}$ and $\mathrm{NCH}_{3}$ signals are relatively broad compared with those observed for the activated complexes of $\mathbf{1 - 6}$. The analyses of the ${ }^{1} \mathrm{H}$ NMR spectra recorded on the activation reactions of $\mathbf{1 0}$ and $\mathbf{1 1}$ indicated that the desired ion-pair complexes were also generated by the action of $\left[\left(\mathrm{C}_{18} \mathrm{H}_{37}\right)_{2} \mathrm{~N}(\mathrm{H}) \mathrm{Me}\right]^{+}\left[\mathrm{B}\left(\mathrm{C}_{6} \mathrm{~F}_{5}\right)_{4}\right]^{-}$(Figures S21 and S22). However, in those cases, the $\mathrm{NCH}_{2}$ and $\mathrm{NCH}_{3}$ signals are much broader than in the case of $\mathbf{1 2}$, although the signals assigned to the ligand framework and $\mathrm{Hf}-\mathrm{CH}_{3}$ are similarly sharp. For 15 and 16, which bear naphthyl groups, some solid was precipitated when they were reacted with $\left[\left(\mathrm{C}_{18} \mathrm{H}_{37}\right)_{2} \mathrm{~N}(\mathrm{H}) \mathrm{Me}\right]^{+}\left[\mathrm{B}\left(\mathrm{C}_{6} \mathrm{~F}_{5}\right)_{4}\right]^{-}$in $\mathrm{C}_{6} \mathrm{D}_{6}$. However, the analyses of the ${ }^{1} \mathrm{H}$ NMR spectra of the soluble portion indicated the generation of the desired ion-pair complexes, although the yield was low ( 70\%) (Figures S24 and S25).

\subsection{Polymerization Studies}

The prepared complexes, which were (1-6, 10-12, and 15,16) activated with $\left[\left(\mathrm{C}_{18} \mathrm{H}_{37}\right)_{2} \mathrm{~N}(\mathrm{H})\right.$ $\mathrm{Me}]^{+}\left[\mathrm{B}\left(\mathrm{C}_{6} \mathrm{~F}_{5}\right)_{4}\right]^{-}$, were screened for ethylene/propylene copolymerization in methylcyclohexane, at an initial temperature of $80^{\circ} \mathrm{C}$, under 20 bar of ethylene/propylene mixed gas. Half-metallocene hafnium complexes 1-6 were inactive, although the activation reaction with $\left[\left(\mathrm{C}_{18} \mathrm{H}_{37}\right)_{2} \mathrm{~N}(\mathrm{H}) \mathrm{Me}\right]^{+}\left[\mathrm{B}\left(\mathrm{C}_{6} \mathrm{~F}_{5}\right)_{4}\right]^{-}$ cleanly generated the desired ion-pair complexes. Complexes 10, 11, and 12 were active and their activity was increased as the increase in the steric bulkiness of the attached substituent and the highest activity was observed with $\mathbf{1 2}$ bearing an isopropyl substituent (entry 3 in Table 1). However, it did not compete with the prototype Dow catalyst III; the productivity of $\mathbf{1 2}$ was $\sim$ half of III (entry 3 vs. 5). For the prototype catalysts, III, which bears a naphthyl group, exhibited higher activity than its analogue, bearing a phenyl substituent. However, in our case, replacing the phenyl group with naphthyl resulted in lowered activity (i.e., 12 vs. 16; entry 3 vs. 4). The Hf-C bonding is significantly ionic and the bonding energy may be sensitive to the steric congestion [49]. When the 
steric congestion is insignificant, the ionic Hf-C bond becomes strong and the olefin insertion through it may be less favorable, leading to lowered activity. We hypothesized that the Hf center in $\mathbf{1 2}$ is not as sterically congested as it is in III. Typically, the steric hindrance around the metal center influences the comonomer incorporation; the more widely opened the reaction site, the higher the incorporation of $\alpha$-olefin. However, the prototype Dow catalyst III is exceptional in the incorporation of high amount of $\alpha$-olefin, regardless of the sterical congestion of the reaction site. Whereas III was able to incorporate $56 \mathrm{~mol} \%$ of propylene, 10-12 and $\mathbf{1 6}$ incorporated only 10-13 mol \% of propylene under the same reaction condition. Accordingly, the polymers generated by III are amorphous, while the polymers generated by $\mathbf{1 0 - 1 2}$ and 16 exhibited melting signals around $100^{\circ} \mathrm{C}$. We also reported various type of $\mathrm{Hf}$ complexes $\left([\mathrm{N}, \mathrm{P}] \mathrm{Hf}\left(\mathrm{CH}_{2} \mathrm{Ph}\right)_{3},[\mathrm{~N}, \mathrm{P}, \mathrm{N}] \mathrm{HfMe}{ }_{2}\right.$, and $[\mathrm{N}, \mathrm{N}] \mathrm{Hf}\left(\mathrm{CH}_{2} \mathrm{Ph}\right)_{3}$-type) with tetrahydroquinoline and tetrahydrophenanthroline frameworks, which were also inferior to III in terms of the $\alpha$-olefin incorporation capability as well as the activity [50-52].

A higher molecular weight polymer was generated with 12 relative to III $\left(M_{n}, 124 \mathrm{kDa}\right.$ vs. $\left.61 \mathrm{kDa}\right)$. The $M_{n}$ value of the generated polymer was increased further to $190 \mathrm{kDa}$ by replacing trioctylaluminum with MMAO, which was employed as a scavenger. These results indicated that trioctylaluminum was engaged in the chain transfer process through the alkyl exchange between $\mathrm{Al}$ and the chain-growing Hf centers, leading to a lowered molecular weight, and that such chain transfer reactions could be suppressed by employing MMAO instead of trioctylaluminum as a scavenger. The unique advantage of III over the other types of catalysts is that it can be used in CCTP, which is performed in the presence of chain transfer agent $\left(\mathrm{R}_{2} \mathrm{Zn}\right)$ deliberately added. III is capable of generating PO chains attached on $\mathrm{Zn}$ sites (i.e., (polyolefinyl) ${ }_{2} \mathrm{Zn}$ ) with negligible a $\beta$-elimination process, which process is inevitable with the conventional metallocene and half-metallocene catalysts. When the polymerizations were performed with 12 in the presence of (octyl $)_{2} \mathrm{Zn}$, which was deliberately added as a chain transfer agent, the $M_{n}$ values were sensitive to the amount of $(\text { octyl })_{2} Z n$ and the observed $M_{n}$ values after the universal calibration (i.e., converted by the equation ' $M_{\mathrm{PO}}=0.495 \times M_{\mathrm{PS}}{ }^{0.990} /(1-\mathrm{S})^{\prime}$, where $\mathrm{S}$ is the mass fraction of the $\mathrm{CH}_{3}$-side chains, i.e., $\left.\mathrm{S}=\left(15 \times\left[\mathrm{C}_{3} \mathrm{H}_{6}\right]\right) /\left[\left(1-\left[\mathrm{C}_{3} \mathrm{H}_{6}\right]\right) \times 28+\left(\left[\mathrm{C}_{3} \mathrm{H}_{6}\right] \times 42\right)\right]\right)[11]$ were in good agreement with the expected values, calculated based on the amount of (octyl $)_{2} \mathrm{Zn}$ employed and the amount of generated polymer $\left(M_{\mathrm{n}}{ }^{\text {expected }}=\right.$ yield $\left.(\mathrm{g}) /(2 \times \mathrm{Zn}-\mathrm{mol})\right) \cdot\left(M_{\mathrm{n}}{ }^{\text {PO-equivalent }}=30,17,12\right.$, $5.9 \mathrm{kDa}$ vs. $M_{\mathrm{n}}{ }^{\text {expected }}=32,17,9.0,4.5$, respectively; entries 8-11; Figure 4). These results indicated that 12 worked well in CCTP, successfully converting the (octyl) $2 \mathrm{Zn}$ to (polyolefinyl) ${ }_{2} \mathrm{Zn}$ with a negligible $\beta$-elimination process, although the activity and the capability for $\alpha$-olefin incorporation were inferior compared to those of III.

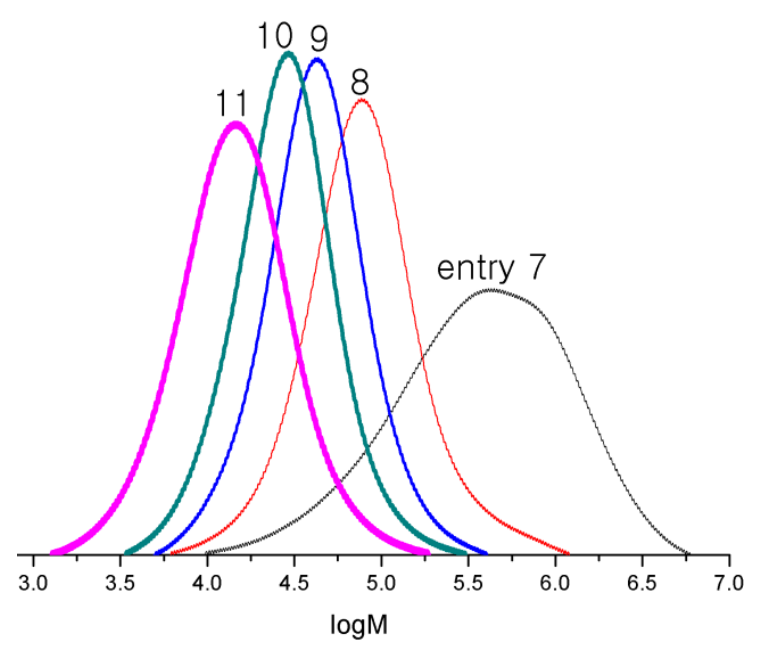

Figure 4. GPC curves for the polymers obtained in CCTP. 
Table 1. Polymerization results ${ }^{\mathrm{a}}$.

\begin{tabular}{|c|c|c|c|c|c|c|c|c|}
\hline Entry & Catalyst & $\begin{array}{c}\mathrm{Al}^{\mathrm{b}} \\
(50 \mu \mathrm{mol})\end{array}$ & $\begin{array}{c}\mathrm{Zn}^{\mathrm{c}} \\
(\mu \mathrm{mol})\end{array}$ & $\begin{array}{l}\text { Yield } \\
\text { (g) }\end{array}$ & $\begin{array}{c}{\left[\mathrm{C}_{3} \mathrm{H}_{6}\right]^{\mathrm{d}}} \\
(\mathrm{mol} \%)\end{array}$ & $\begin{array}{l}T_{\mathrm{m}} \\
\left({ }^{\circ} \mathrm{C}\right)\end{array}$ & $\begin{array}{l}M_{\mathrm{n}} \mathrm{e} \\
(\mathrm{kDa})\end{array}$ & $M_{\mathrm{w}} / M_{\mathrm{n}}$ \\
\hline 1 & 10 & TOA & 0 & 1.2 & 10 & $86-129$ & 16 & 11.0 \\
\hline 2 & 11 & TOA & 0 & 5.8 & 12 & $101-120$ & 87 & 1.5 \\
\hline 3 & 12 & TOA & 0 & 7.5 & 13 & $102-120$ & 124 & 3.2 \\
\hline 4 & 16 & TOA & 0 & 3.5 & 11 & 99-115 & 26 & 2.0 \\
\hline 5 & III & TOA & 0 & 16 & 56 & not-detected & 61 & 2.6 \\
\hline 6 & 11 & MMAO & 0 & 5.5 & 10 & $105-120$ & 266 & 2.1 \\
\hline 7 & 12 & MMAO & 0 & 7.8 & 11 & $102-117$ & 190 & 3.4 \\
\hline 8 & 12 & MMAO & 100 & 6.3 & 9.0 & $100-117$ & $58(30 ; 32)^{\mathrm{f}}$ & 1.8 \\
\hline 9 & 12 & MMAO & 200 & 6.6 & 11 & $100-112$ & $33(17 ; 17)^{f}$ & 1.6 \\
\hline 10 & 12 & MMAO & 300 & 5.4 & 13 & $97-110$ & $22(12 ; 9.0)^{\mathrm{f}}$ & 1.6 \\
\hline 11 & 12 & MMAO & 400 & 3.6 & 15 & 94-108 & $11(5.9 ; 4.5)^{\mathrm{f}}$ & 1.8 \\
\hline
\end{tabular}

${ }^{a}$ Polymerization conditions: Hf complex $(2.0 \mu \mathrm{mol})$, activator $\left(\left[\left(\mathrm{C}_{18} \mathrm{H}_{37}\right)_{2} \mathrm{~N}(\mathrm{H}) \mathrm{Me}\right]^{+}\left[\mathrm{B}\left(\mathrm{C}_{6} \mathrm{~F}_{5}\right)_{4}\right]^{-}, 2.0 \mu \mathrm{mol}\right)$, methylcyclohexane $(26 \mathrm{~g})$, ethylene and propylene mixed gas $(1: 1,20 \mathrm{bar}), 80-90^{\circ} \mathrm{C} .50 \mathrm{~min} .{ }^{\mathrm{b}} \mathrm{TOA}$ (trioctylaluminum) or MMAO (modified-methylaluminoxane) was employed as a scavenger. ${ }^{c}(\mathrm{Octyl}){ }_{2} \mathrm{Zn}$ was employed as a chain transfer agent. ${ }^{\mathrm{d}}$ Propylene content measured by ${ }^{1} \mathrm{H}$-NMR spectra. e Measured by GPC at $160{ }^{\circ} \mathrm{C}$, using trichlorobenzene and calculated relative to PS standards. ${ }^{\mathrm{f}}$ PO-equivalent value converted by the equation $' M_{\mathrm{PO}}=0.495 \times M_{\mathrm{PS}}{ }^{0.990} /(1-\mathrm{S})^{\prime}$, where $\mathrm{S}$ is the mass fraction of the $\mathrm{CH}_{3}$-side chains, i.e., $\mathrm{S}=\left(15 \times\left[\mathrm{C}_{3} \mathrm{H}_{6}\right]\right) /$ $\left[\left(1-\left[\mathrm{C}_{3} \mathrm{H}_{6}\right]\right) \times 28+\left(\left[\mathrm{C}_{3} \mathrm{H}_{6}\right] \times 42\right)\right]$ and the expected value calculated by 'yield $(\mathrm{g}) /(2 \times \mathrm{Zn}-\mathrm{mol})^{\prime}$ ' are written in the parenthesis.

\section{Conclusions}

A series of half metallocene $\mathrm{HfMe}_{2}$ complexes bearing a tetrahydroquinoline or tetrahydroquinaldine framework and tetramethylcyclopentadienyl, thiophene-fused dimethylcyclopentadienyl, or fluorenyl ligand, were prepared by sequential treatments of four equiv. $\mathrm{MeMgBr}$ and $\mathrm{HfCl}_{4}$ to the ligand precursors. A series of $\left[\mathrm{N}^{\text {amido }}, \mathrm{N}, \mathrm{C}^{\text {aryl }}\right] \mathrm{HfMe}_{2}$-type post-metallocene complexes bearing a tetrahydrophenanthroline framework, substituted with n-butyl or isopropyl group at position 2 and phenyl or naphthyl group at position 9, were prepared by the treatment of the ligand precursor with in situ generated $\mathrm{HfMe}_{4}$. The structures of many of the prepared complexes were confirmed by $\mathrm{X}$-ray crystallography. The activation reaction of the half metallocene $\mathrm{HfMe}_{2}$ complexes with $\left[\left(\mathrm{C}_{18} \mathrm{H}_{37}\right)_{2} \mathrm{~N}(\mathrm{H}) \mathrm{Me}\right]^{+}\left[\mathrm{B}\left(\mathrm{C}_{6} \mathrm{~F}_{5}\right)_{4}\right]^{-}$ cleanly afforded the desired ion-pair complexes, although the reaction was slow. However, the activated complexes were inactive in ethylene/propylene copolymerization. The activated complexes, generated from post-metallocene $\mathrm{HfMe}_{2}$ complexes by the action of $\left.\left[\left(\mathrm{C}_{18} \mathrm{H}_{37}\right)_{2} \mathrm{~N}(\mathrm{H}) \mathrm{Me}\right)\right]^{+}\left[\mathrm{B}\left(\mathrm{C}_{6} \mathrm{~F}_{5}\right)_{4}\right]^{-}$were active. The highest activity was observed with $\mathbf{1 2}$, which bears a bulky isopropyl group. However, $\mathbf{1 2}$ was inferior to the prototype pyridylamido-Hf Dow catalyst (III) in terms of the activity and $\alpha$-olefin incorporation capability. Furthermore, 12 performed well in the CCTP, which was performed in the presence of (octyl) ${ }_{2} \mathrm{Zn}$, converting the (octyl) ${ }_{2} \mathrm{Zn}$ to (polyolefinyl) ${ }_{2} \mathrm{Zn}$ with controlled lengths of the polyolefinyl chain.

Supplementary Materials: The following are available online at http:/www.mdpi.com/2073-4360/11/7/1093/s1, Figures S1-S14: ${ }^{1} \mathrm{H}$ and ${ }^{13} \mathrm{C}$ NMR spectra of 1-6 and 9-16; Figures S15-S26: ${ }^{1} \mathrm{H}$ NMR spectra of the activated complexes with $\left[\left(\mathrm{C}_{18} \mathrm{H}_{37}\right)_{2} \mathrm{~N}(\mathrm{H}) \mathrm{Me}\right]^{+}\left[\mathrm{B}\left(\mathrm{C}_{6} \mathrm{~F}_{5}\right)_{4}\right]^{-}$; Figures $\mathrm{S} 27$ and S28: ${ }^{1} \mathrm{H}$ NMR spectra of the anhydrous and the water-containing $\left[\left(\mathrm{C}_{18} \mathrm{H}_{37}\right)_{2} \mathrm{~N}(\mathrm{H}) \mathrm{Me}\right]^{+}\left[\mathrm{B}\left(\mathrm{C}_{6} \mathrm{~F}_{5}\right)_{4}\right]^{-}$; Figures $\mathrm{S} 29$ and S30: ${ }^{1} \mathrm{H}$ NMR spectra of polymers; Figure S31: DSC thermograms.

Author Contributions: B.Y.L., E.J.S., and K.S.L. conceived and designed the experiments; J.W.B., S.J.K., and H.J.L. synthesized the complexes; J.W.B. and T.J.K. performed the polymerizations; J.Y.R. and J.L. studied X-ray crystallography.

Funding: This work was supported by LG and by the Commercialization Promotion Agency for R\&D Outcomes (COMPA) funded by the Ministry of Science and ICT (MSIT) and by a grant from Priority Research Centers Program (2019R1A6A1A11051471) funded by the National Research Foundation of Korea (NRF).

Conflicts of Interest: The authors declare no conflict of interest. 


\section{References}

1. Kaminsky, W. Discovery of Methylaluminoxane as Cocatalyst for Olefin Polymerization. Macromolecules 2012, 45, 3289-3297. [CrossRef]

2. Lee, S.; Park, S.S.; Kim, J.G.; Kim, C.S.; Lee, B.Y. Preparation of "Constrained geometry" titanium complexes of [1,2]azasilinane framework for ethylene/1-octene copolymerization. Molecules 2017, 22, 258. [CrossRef] [PubMed]

3. Makio, H.; Terao, H.; Iwashita, A.; Fujita, T. FI catalysts for olefin polymerization-A comprehensive treatment. Chem. Rev. 2011, 111, 2363-2449. [CrossRef] [PubMed]

4. Cano, J.; Kunz, K. How to synthesize a constrained geometry catalyst (CGC)—A survey. J. Organomet. Chem. 2007, 692, 4411-4423. [CrossRef]

5. Arriola, D.J.; Carnahan, E.M.; Hustad, P.D.; Kuhlman, R.L.; Wenzel, T.T. Catalytic production of olefin block copolymers via chain shuttling polymerization. Science 2006, 312, 714-719. [CrossRef]

6. Boussie, T.R.; Diamond, G.M.; Goh, C.; Hall, K.A.; LaPointe, A.M.; Leclerc, M.K.; Murphy, V.; Shoemaker, J.A.W.; Turner, H.; Rosen, R.K.; et al. Nonconventional Catalysts for Isotactic Propene Polymerization in Solution Developed by Using High-Throughput-Screening Technologies. Angew. Chem. Int. Ed. 2006, 45, 3278-3283. [CrossRef] [PubMed]

7. Frazier, K.A.; Froese, R.D.; He, Y.; Klosin, J.; Theriault, C.N.; Vosejpka, P.C.; Zhou, Z.; Abboud, K.A. Pyridylamido hafnium and zirconium complexes: Synthesis, dynamic behavior, and ethylene/1-octene and propylene polymerization reactions. Organometallics 2011, 30, 3318-3329. [CrossRef]

8. Alfano, F.; Boone, H.W.; Busico, V.; Cipullo, R.; Stevens, J.C. Polypropylene "Chain Shuttling" at Enantiomorphous and Enantiopure Catalytic Species: Direct and Quantitative Evidence from Polymer Microstructure. Macromolecules 2007, 40, 7736-7738. [CrossRef]

9. Domski, G.J.; Eagan, J.M.; De Rosa, C.; Di Girolamo, R.; LaPointe, A.M.; Lobkovsky, E.B.; Talarico, G.; Coates, G.W. Combined Experimental and Theoretical Approach for Living and Isoselective Propylene Polymerization. ACS Catal. 2017, 7, 6930-6937. [CrossRef]

10. De Rosa, C.; Di Girolamo, R.; Talarico, G. Expanding the Origin of Stereocontrol in Propene Polymerization Catalysis. ACS Catal. 2016, 6, 3767-3770. [CrossRef]

11. Park, S.S.; Kim, C.S.; Kim, S.D.; Kwon, S.J.; Lee, H.M.; Kim, T.H.; Jeon, J.Y.; Lee, B.Y. Biaxial Chain Growth of Polyolefin and Polystyrene from 1,6-Hexanediylzinc Species for Triblock Copolymers. Macromolecules 2017, 50, 6606-6616. [CrossRef]

12. Leclerc, M.K.; Brintzinger, H.H. Origins of stereoselectivity and stereoerror formation in ansa-zirconocene-catalyzed isotactic propene polymerization. A deuterium labeling study. J. Am. Chem. Soc. 1995, 117, 1651-1652. [CrossRef]

13. Eagan, J.M.; Xu, J.; Di Girolamo, R.; Thurber, C.M.; Macosko, C.W.; La Pointe, A.M.; Bates, F.S.; Coates, G.W. Combining polyethylene and polypropylene: Enhanced performance with PE/iPP multiblock polymers. Science 2017, 355, 814-816. [CrossRef] [PubMed]

14. Valente, A.; Mortreux, A.; Visseaux, M.; Zinck, P. Coordinative chain transfer polymerization. Chem. Rev. 2013, 113, 3836-3857. [CrossRef] [PubMed]

15. van Meurs, M.; Britovsek, G.J.P.; Gibson, V.C.; Cohen, S.A. Polyethylene Chain Growth on Zinc Catalyzed by Olefin Polymerization Catalysts: A Comparative Investigation of Highly Active Catalyst Systems across the Transition Series. J. Am. Chem. Soc. 2005, 127, 9913-9923. [CrossRef] [PubMed]

16. Rocchigiani, L.; Busico, V.; Pastore, A.; Macchioni, A. Comparative NMR Study on the Reactions of Hf(IV) Organometallic Complexes with Al/Zn Alkyls. Organometallics 2016, 35, 1241-1250. [CrossRef]

17. Hustad, P.O.; Kuhlman, R.L.; Arriola, D.J.; Carnahan, E.M.; Wenzel, T.T. Continuous production of ethylene-based diblock copolymers using coordinative chain transfer polymerization. Macromolecules 2007, 40, 7061-7064. [CrossRef]

18. Kim, S.D.; Kim, T.J.; Kwon, S.J.; Kim, T.H.; Baek, J.W.; Park, H.S.; Lee, H.J.; Lee, B.Y. Peroxide-Mediated Alkyl-Alkyl Coupling of Dialkylzinc: A Useful Tool for Synthesis of ABA-Type Olefin Triblock Copolymers. Macromolecules 2018, 51, 4821-4828. [CrossRef]

19. Vittoria, A.; Busico, V.; Cannavacciuolo, F.D.; Cipullo, R. Molecular Kinetic Study of "Chain Shuttling" Olefin Copolymerization. ACS Catal. 2018, 8, 5051-5061. [CrossRef] 
20. Jeon, J.Y.; Park, S.H.; Kim, D.H.; Park, S.S.; Park, G.H.; Lee, B.Y. Synthesis of polyolefin-block-polystyrene through sequential coordination and anionic polymerizations. J. Polym. Sci. Part A Polym. Chem. 2016, 54, 3110-3118. [CrossRef]

21. Kim, C.S.; Park, S.S.; Kim, S.D.; Kwon, S.J.; Baek, J.W.; Lee, B.Y. Polystyrene chain growth from di-end-functional polyolefins for polystyrene-polyolefin-polystyrene block copolymers. Polymers 2017, 9, 481. [CrossRef] [PubMed]

22. Kim, D.H.; Park, S.S.; Park, S.H.; Jeon, J.Y.; Kim, H.B.; Lee, B.Y. Preparation of polystyrene-polyolefin multiblock copolymers by sequential coordination and anionic polymerization. RSC Adv. 2017, 7, 5948-5956. [CrossRef]

23. Liu, C.-C.; Liu, Q.; Lo, P.-K.; Lau, K.-C.; Yiu, S.-M.; Chan, M.C.W. Olefin Polymerization Reactivity of Group 4 Post-Metallocene Catalysts Bearing a Four-Membered C(sp $\left.{ }^{3}\right)$-Donor Chelate Ring. ChemCatChem 2019, 11, 628-635. [CrossRef]

24. Kwon, S.J.; Baek, J.W.; Lee, H.J.; Kim, T.J.; Ryu, J.Y.; Lee, J.; Shin, E.J.; Lee, K.S.; Lee, B.Y. Preparation of Pincer Hafnium Complexes for Olefin Polymerization. Molecules 2019, 24, 1676. [CrossRef] [PubMed]

25. Matsumoto, K.; Takayanagi, M.; Suzuki, Y.; Koga, N.; Nagaoka, M. Atomistic chemical computation of Olefin polymerization reaction catalyzed by (pyridylamido)hafnium(IV) complex: Application of Red Moon simulation. J. Comput. Chem. 2019, 40, 421-429. [CrossRef] [PubMed]

26. Schnee, G.; Farenc, M.; Bitard, L.; Vantomme, A.; Welle, A.; Brusson, J.M.; Afonso, C.; Giusti, P.; Carpentier, J.F.; Kirillov, E. Synthesis, APPI mass-spectrometric characterization, and polymerization studies of group 4 dinuclear bis(Ansa-metallocene) complexes. Catalysts 2018, 8, 558. [CrossRef]

27. Xu, J.; Eagan, J.M.; Kim, S.-S.; Pan, S.; Lee, B.; Klimovica, K.; Jin, K.; Lin, T.-W.; Howard, M.J.; Ellison, C.J.; et al. Compatibilization of Isotactic Polypropylene (iPP) and High-Density Polyethylene (HDPE) with iPP-PE Multiblock Copolymers. Macromolecules 2018, 51, 8585-8596. [CrossRef]

28. Zhang, J.; Motta, A.; Gao, Y.; Stalzer, M.M.; Delferro, M.; Liu, B.; Lohr, T.L.; Marks, T.J. Cationic Pyridylamido Adsorbate on Brønsted Acidic Sulfated Zirconia: A Molecular Supported Organohafnium Catalyst for Olefin Homo- and Co-Polymerization. ACS Catal. 2018, 8, 4893-4901. [CrossRef]

29. Cueny, E.S.; Johnson, H.C.; Landis, C.R. Selective Quench-Labeling of the Hafnium-Pyridyl Amido-Catalyzed Polymerization of 1-Octene in the Presence of Trialkyl-Aluminum Chain-Transfer Reagents. ACS Catal. 2018, 8, 11605-11614. [CrossRef]

30. Johnson, H.C.; Cueny, E.S.; Landis, C.R. Chain Transfer with Dialkyl Zinc during Hafnium-Pyridyl Amido-Catalyzed Polymerization of 1-Octene: Relative Rates, Reversibility, and Kinetic Models. ACS Catal. 2018, 8, 4178-4188. [CrossRef]

31. Gao, Y.; Chen, X.; Zhang, J.; Chen, J.; Lohr, T.L.; Marks, T.J. Catalyst Nuclearity Effects on Stereo- and Regioinduction in Pyridylamidohafnium-Catalyzed Propylene and 1-Octene Polymerizations. Macromolecules 2018, 51, 2401-2410. [CrossRef]

32. Matsumoto, K.; Takayanagi, M.; Sankaran, S.K.; Koga, N.; Nagaoka, M. Role of the Counteranion in the Reaction Mechanism of Propylene Polymerization Catalyzed by a (Pyridylamido)hafnium(IV) Complex. Organometallics 2018, 37, 343-349. [CrossRef]

33. Wu, C.J.; Lee, S.H.; Yun, H.; Lee, B.Y. Ortho lithiation of tetrahydroquinoline derivatives and its use for the facile construction of polymerization catalysts. Organometallics 2007, 26, 6685-6687. [CrossRef]

34. Park, J.H.; Do, S.H.; Cyriac, A.; Yun, H.; Lee, B.Y. Preparation of half-metallocenes of thiophene-fused and tetrahydroquinoline-linked cyclopentadienyl ligands for ethylene/ $\alpha$-olefin copolymerization. Dalton Trans. 2010, 39, 9994-10002. [CrossRef] [PubMed]

35. Wang, T.; Chen, F.; Qin, J.; He, Y.-M.; Fan, Q.-H. Asymmetric Ruthenium-Catalyzed Hydrogenation of 2- and 2,9-Substituted 1,10-Phenanthrolines. Angew. Chem. Int. Ed. 2013, 52, 7172-7176. [CrossRef] [PubMed]

36. Robert, K.R.; VanderLende, D.D. Highly soluble olefin polymerization catalyst activator. U.S. Patent 5,919,983, 6 July 1999.

37. Cho, D.J.; Wu, C.J.; Sujith, S.; Han, W.S.; Kang, S.O.; Lee, B.Y. o-phenylene-bridged Cp/amido titanium complexes for ethylene/1-hexene copolymerizations. Organometallics 2006, 25, 2133-2134. [CrossRef]

38. Wu, C.J.; Lee, S.H.; Yu, S.T.; Na, S.J.; Yun, H.; Lee, B.Y. $\mathrm{CO}_{2}$-mediated ortho-lithiatium of N-alkylanilines and its use for the construction of polymerization catalysts. Organometallics 2008, 27, 3907-3917. [CrossRef] 
39. Kim, S.; Park, J.; Song, B.; Yoon, S.-W.; Go, M.; Lee, J.; Lee, B. Preparation of Thiophene-Fused and Tetrahydroquinoline-Linked Cyclopentadienyl Titanium Complexes for Ethylene/ $\alpha$-Olefin Copolymerization. Catalysts 2013, 3, 104-124. [CrossRef]

40. Resconi, L.; Camurati, I.; Grandini, C.; Rinaldi, M.; Mascellani, N.; Traverso, O. Indenyl-amido titanium and zirconium dimethyl complexes: improved synthesis and use in propylene polymerization. J. Organomet. Chem. 2002, 664, 5-26. [CrossRef]

41. Okuda, J.; Amor, F.; du Plooy, K.E.; Eberle, T.; Hultzsch, K.C.; Spaniol, T.P. Zirconium, hafnium and yttrium complexes containing two linked amido—-tetramethylcyclopentadienyl ligands: Synthesis, reactivity and molecular structure of $\mathrm{Hf}\left(\eta^{5}: \eta^{1}-\mathrm{C}_{5} \mathrm{Me}_{4} \mathrm{SiMe}_{2} \mathrm{NiPr}\right)_{2}$. Polyhedron 1998, 17, 1073-1080. [CrossRef]

42. Carpenetti, D.W.; Kloppenburg, L.; Kupec, J.T.; Petersen, J.L. Application of Amine Elimination for the Efficient Preparation of Electrophilic ansa-Monocyclopentadienyl Group 4 Complexes Containing an Appended Amido Functionality. Structural Characterization of $\left[\left(\mathrm{C}_{5} \mathrm{H}_{4}\right) \mathrm{SiMe}_{2}(\mathrm{~N}-\mathrm{t}-\mathrm{Bu})\right] \mathrm{ZrCl}_{2}\left(\mathrm{NMe}_{2} \mathrm{H}\right)$. Organometallics 1996, 15, 1572-1581. [CrossRef]

43. Sun, Y.; Xu, B.; Shiono, T.; Cai, Z. Highly Active ansa-(Fluorenyl)(amido)titanium-Based Catalysts with Low Load of Methylaluminoxane for Syndiotactic-Specific Living Polymerization of Propylene. Organometallics 2017, 36, 3009-3012. [CrossRef]

44. Liu, C.-C.; Chan, M.C.W. Chelating $\sigma$-Aryl Post-Metallocenes: Probing Intramolecular [C-H…F-C] Interactions and Unusual Reaction Pathways. Acc. Chem. Res. 2015, 48, 1580-1590. [CrossRef] [PubMed]

45. Froese, R.D.J.; Hustad, P.D.; Kuhlman, R.L.; Wenzel, T.T. Mechanism of Activation of a Hafnium Pyridyl-Amide Olefin Polymerization Catalyst: Ligand Modification by Monomer. J. Am. Chem. Soc. 2007, 129, 7831-7840. [CrossRef] [PubMed]

46. Zhang, C.; Pan, H.; Klosin, J.; Tu, S.; Jaganathan, A.; Fontaine, P.P. Synthetic Optimization and Scale-Up of Imino-Amido Hafnium and Zirconium Olefin Polymerization Catalysts. Org. Proc. Res. Dev. 2015, 19, 1383-1391. [CrossRef]

47. Cueny, E.S.; Johnson, H.C.; Anding, B.J.; Landis, C.R. Mechanistic Studies of Hafnium-Pyridyl Amido-Catalyzed 1-Octene Polymerization and Chain Transfer Using Quench-Labeling Methods. J. Am. Chem. Soc. 2017, 139, 11903-11912. [CrossRef] [PubMed]

48. Zuccaccia, C.; Macchioni, A.; Busico, V.; Cipullo, R.; Talarico, G.; Alfano, F.; Boone, H.W.; Frazier, K.A.; Hustad, P.D.; Stevens, J.C.; et al. Intra- and Intermolecular NMR Studies on the Activation of Arylcyclometallated Hafnium Pyridyl-Amido Olefin Polymerization Precatalysts. J. Am. Chem. Soc. 2008, 130, 10354-10368. [CrossRef] [PubMed]

49. Machat, M.R.; Fischer, A.; Schmitz, D.; Vöst, M.; Drees, M.; Jandl, C.; Pöthig, A.; Casati, N.P.M.; Scherer, W.; Rieger, B. Behind the Scenes of Group 4 Metallocene Catalysis: Examination of the Metal-Carbon Bond. Organometallics 2018, 37, 2690-2705. [CrossRef]

50. Lee, C.S.; Park, J.H.; Hwang, E.Y.; Park, G.H.; Go, M.J.; Lee, J.; Lee, B.Y. Preparation of [Bis(amido)-phosphine] and [Amido-Phosphine Sulfide or Oxide] Hafnium and Zirconium Complexes for Olefin Polymerization. J. Organom. Chem. 2014, 772, 172-181. [CrossRef]

51. Hwang, E.Y.; Park, G.H.; Lee, C.S.; Kang, Y.Y.; Lee, J.; Lee, B.Y. Preparation of octahydro- and tetrahydro-[1,10] phenanthroline zirconium and hafnium complexes for olefin polymerization. Dalton Trans. 2015, 44, 3845-3855. [CrossRef] [PubMed]

52. Jun, S.H.; Park, J.H.; Lee, C.S.; Park, S.Y.; Go, M.J.; Lee, J.; Lee, B.Y. Preparation of phosphine-amido hafnium and zirconium complexes for olefin polymerization. Organometallics 2013, 32, 7357-7365. [CrossRef]

(C) 2019 by the authors. Licensee MDPI, Basel, Switzerland. This article is an open access article distributed under the terms and conditions of the Creative Commons Attribution (CC BY) license (http://creativecommons.org/licenses/by/4.0/). 